\title{
Nicotinamide mononucleotide requires SIRT3 to improve cardiac function and bioenergetics in a Friedreich's ataxia cardiomyopathy model
}

Angelical S. Martin,, ${ }^{1,2}$ Dennis M. Abraham, ${ }^{3}$ Kathleen A. Hershberger, ${ }^{1,2}$ Dhaval P. Bhatt, ${ }^{1}$ Lan Mao, ${ }^{3}$ Huaxia Cui, ${ }^{1}$ Juan Liu, ${ }^{2}$ Xiaojing Liu, ${ }^{2}$ Michael J. Muehlbauer, ${ }^{1}$ Paul A. Grimsrud, ${ }^{1}$ Jason W. Locasale, ${ }^{1,2}$ R. Mark Payne, ${ }^{4}$ and Matthew D. Hirschey ${ }^{1,2,5}$

'Duke Molecular Physiology Institute and Sarah W. Stedman Nutrition and Metabolism Center, ${ }^{2}$ Department of Pharmacology and Cancer Biology, ${ }^{3}$ Department of Medicine, Division of Cardiology and Duke Cardiovascular Physiology Core, Duke University Medical Center, Durham, North Carolina, USA. ${ }^{4}$ Department of Medicine, Division of Pediatrics, Indiana University, Indianapolis, Indiana, USA. ${ }^{5}$ Department of Medicine, Division of Endocrinology, Metabolism, \& Nutrition, Duke University Medical Center, Durham, North Carolina, USA.

Increasing $\mathrm{NAD}^{+}$levels by supplementing with the precursor nicotinamide mononucleotide (NMN) improves cardiac function in multiple mouse models of disease. While NMN influences several aspects of mitochondrial metabolism, the molecular mechanisms by which increased NAD+ enhances cardiac function are poorly understood. A putative mechanism of NAD+ therapeutic action exists via activation of the mitochondrial $N A D^{+}$-dependent protein deacetylase sirtuin 3 (SIRT3). We assessed the therapeutic efficacy of NMN and the role of SIRT3 in the Friedreich's ataxia cardiomyopathy mouse model (FXN-KO). At baseline, the FXN-KO heart has mitochondrial protein hyperacetylation, reduced Sirt3 mRNA expression, and evidence of increased NAD+ salvage. Remarkably, NMN administered to FXN-KO mice restores cardiac function to near-normal levels. To determine whether SIRT3 is required for NMN therapeutic efficacy, we generated SIRT3-KO and SIRT3-KO/FXN-KO (double KO [dKO]) models. The improvement in cardiac function upon NMN treatment in the FXN-KO is lost in the dKO model, demonstrating that the effects of NMN are dependent upon cardiac SIRT3. Coupled with cardio-protection, SIRT3 mediates NMN-induced improvements in both cardiac and extracardiac metabolic function and energy metabolism. Taken together, these results serve as important preclinical data for NMN supplementation or SIRT3 activator therapy in Friedreich's ataxia patients.

Conflict of interest: RMP has intellectual property, income, research support, and ownership in a company related to gene therapy in Friedreich's ataxia.

Submitted: March 22, 2017

Accepted: June 6, 2017

Published: July 20, 2017

Reference information: JCI Insight. 2017;2(14):e93885. https://doi.org/10.1172/jci. insight. 93885.

\section{Introduction}

Clinical interest in $\mathrm{NAD}^{+}$precursor supplementation is growing (1-4), which is driven by studies showing therapeutic benefit in several preclinical cardiomyopathy models (for a recent review, see ref. 5). In these models, $\mathrm{NAD}^{+}$precursor supplementation attenuates cardiomyopathy in mitochondrial complex I deficiency (6), extends lifespan in mice with cardiac iron deficiency from genetic ablation of transferrin receptor (7), protects against ischemia-reperfusion (I/R) injury induced ROS formation (8), and blunts development of chronic catecholamine-induced cardiac dysfunction (9). The molecular mechanisms underlying the cardio-protective effects of $\mathrm{NAD}^{+}$supplementation are poorly understood, which is an important conceptual barrier to translating $\mathrm{NAD}^{+}$precursor supplementation into clinical practice.

A leading candidate mechanism of $\mathrm{NAD}^{+}$therapeutic activity involves activation of the $\mathrm{NAD}^{+}$-dependent protein deacylases called sirtuins (SIRTs), specifically the mitochondrial deacetylase SIRT3 (5). SIRT3 is required for both basal cardiac function and stress response. Studies investigating the role of SIRT3 in the heart have found enhanced age-induced cardiac hypertrophy (10) and increased susceptibility to cardiac stressors (9-11) in the absence of SIRT3, likely driven by marked reductions in cardiac ATP levels (12) and mitochondrial protein hyperacetylation (13). Recent work has identified that pathological cardiac remodeling and mitochondrial protein hyperacetylation are linked by redox imbalance (6). Specifically, a decrease 
in the $\mathrm{NAD}^{+} / \mathrm{NADH}$ ratio identified in a mouse model of cardiac complex I deficiency resulted in mitochondrial protein hyperacetylation and was associated with impaired SIRT3 activity. Normalization of the $\mathrm{NAD}^{+} / \mathrm{NADH}$ ratio via nicotinamide mononucleotide (NMN) supplementation alleviated the inhibitory effects of hyperacetylation and attenuated the development of cardiac dysfunction (6). This study, and others $(14,15)$, supports the notion that mitochondrial protein hyperacetylation plays a key role in pathological cardiac dysfunction and, importantly, that treatment targeting this process may be beneficial.

Interestingly, several models of heart failure (HF) are associated with cardiac mitochondrial protein hyperacetylation, as well as human cardiomyopathies $(5,6,15)$, suggesting a fundamental but underappreciated role of mitochondrial protein acetylation and SIRT3 in the pathogenesis of HF. Of interest is the genetic cardiomyopathy model that mimics Friedreich's ataxia (FRDA) cardiomyopathy — the frataxin-KO (Fxn ${ }^{-/-}$, also known as FXN-KO) mouse (16) — which develops mitochondrial protein hyperacetylation (17). FXN is required for energy generation through oxidative metabolism via its effect on iron-sulfur cluster (ISC) assembly. ISC assembly is disrupted in FXN-KO mice, resulting in primary mitochondrial dysfunction due to mitochondrial electron transport chain (ETC) defects that are manifested as both the neuropathology and cardiomyopathy known as FRDA (18). Similar to FRDA patients, the cardiac/skeletal muscle-specific FXN-KO mouse has impaired cardiac oxidative metabolism due to diminished activity of the ETC, which results in energy depletion and HF (16). Altogether, this mouse model develops HF that closely recapitulates the trajectory of disease in humans (19) and displays progressive increases in mitochondrial protein hyperacetylation, which, importantly, can be reversed with induction of Fxn expression via viral gene therapy $(17,20)$. However, major questions remaining in the field include whether protein hyperacetylation contributes to the mitochondrial and metabolic abnormalities in $\mathrm{HF}$ and if targeting SIRT3 activation holds any therapeutic potential. Thus, we set out to determine the efficacy of NMN supplementation in the FXN-KO mouse and to test the role of SIRT3 in mediating the effects of NMN.

\section{Results}

FXN-KO cardiac mitochondria have protein hyperacetylation, redox imbalance, and altered $N A D^{+}$homeostasis. Previous studies show progressive mitochondrial protein hyperacetylation in the FXN-KO heart by Western blotting $(17,20)$. To identify these proteins, we performed isobaric tag-based quantitative proteomics, using well-established methods (21), on mitochondria collected from FXN-KO hearts at 5, 8, and 13 weeks of age (Supplemental Table 1; ProteomeXchange identifier PXD006754). In congruence with the literature, acetyl-proteomic analysis of FXN-KO mitochondria revealed an overall increase in protein hyperacetylation over time (Figure 1A). At 5 weeks, FXN-KO mice were grossly indistinguishable from WT mice (data not shown), and we found no hyperacetylation in the FXN-KO at this time point (Figure 1A, left). As the disease progressed, we found a concomitant increase in acetylation $\left(P_{\text {adjusted }}<0.05\right)$ : 111 and 259 acetyl sites of relative occupancy (i.e., acetyl peptide quantification corrected for change in protein abundance) increasing with greater than $\log _{2}$ fold change ( $\log _{2}$ FC) of 1 (or 2 -fold on an arithmetic scale) at 8 and 13 weeks of age, respectively (Figure 1A, center and right; black dots in pink box). Together, these data show a dramatic and progressive increase in mitochondrial protein acetylation in the hearts of FXN-KO mice.

The primary mitochondrial lesion in FXN-KO mitochondria is due to loss of expression and activity of ISC proteins involved in the ETC (18). Relative protein abundance, as measured by proteomic analyses, of ISC binding proteins and associated subunits in ETC complex I are significantly decreased at 8 and 13 weeks of age as compared with their respective WT controls (Figure 1B, red and dark red). Consequently, complex I activity is greatly impaired (17) resulting in mitochondrial redox imbalance, indicating an elevation in the mitochondrial $\mathrm{NADH} / \mathrm{NAD}^{+}$ratio (22). Indeed, protein abundance of other ETC ISC binding proteins - complex II subunits and complex III Rieske (Supplemental Figure 1, A-D; supplemental material available online with this article; https://doi.org/10.1172/jci.insight.93885DS1) — were also significantly reduced in FXN-KO cardiac mitochondria. Taken together, these data support the idea that an elevated mitochondrial $\mathrm{NADH} / \mathrm{NAD}^{+}$ratio due to the genetic lesion in FXN-KO mice is an important contributor to the mitochondrial protein hyperacetylation in the FXN-KO heart.

Next, we determined if mitochondrial redox imbalances in FXN-KO hearts were associated with changes in the genes and proteins involved in $\mathrm{NAD}^{+}$homeostasis. Indeed, with cardiomyopathies, $\mathrm{NAD}^{+}$ levels can be depleted and de novo synthesis reduced (23-25). In this setting, cardiomyocytes upregulate genes involved in $\mathrm{NAD}^{+}$salvage to compensate for the loss $(14,26)$. Analyses of previously published gene microarrays of cardiac transcripts over the course of disease progression in FXN-KO mice revealed shifts 
A

Age (5, 8, and 13 wks, respectively)

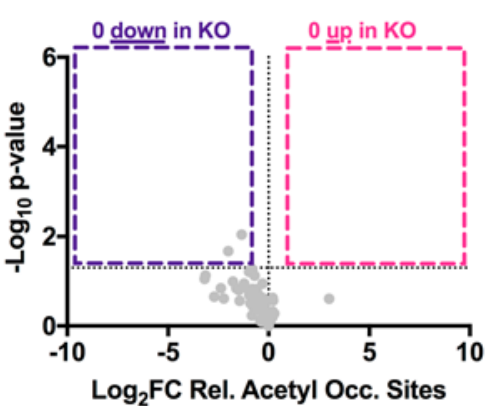

(F/W, 5 wks)

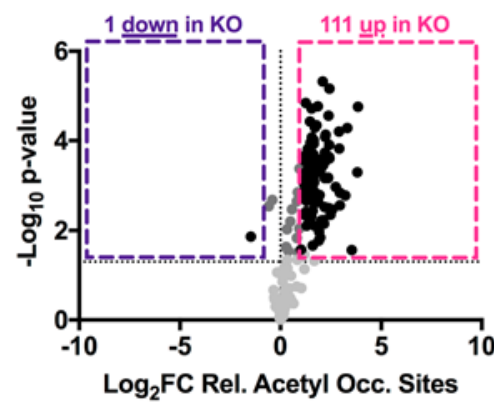

(F/W, 8 wks)

B
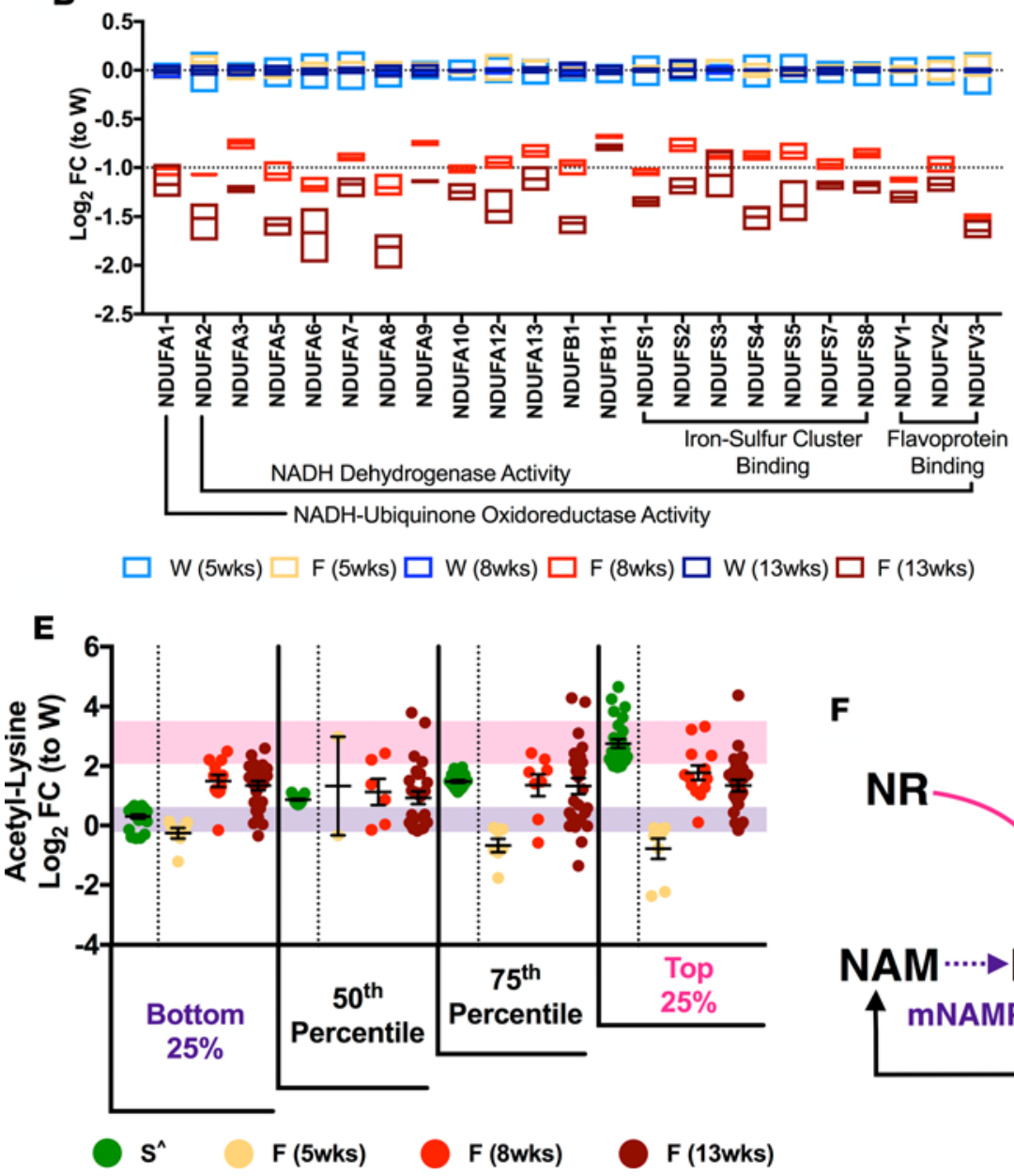

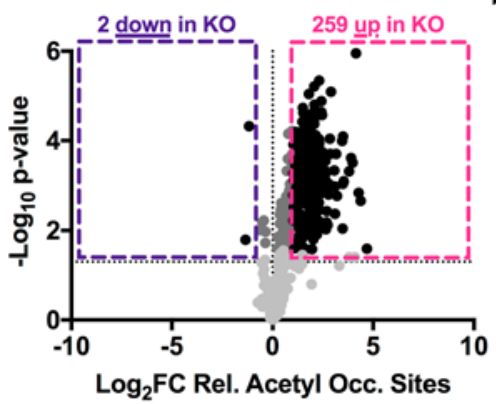

(F/W, 13 wks)

C

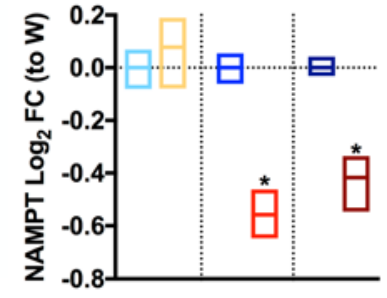

D

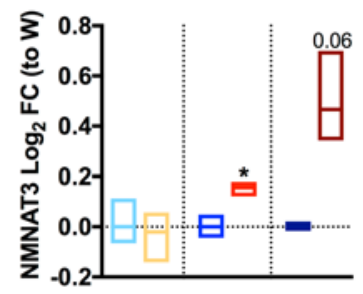
W (5wks)
W (8wks)
W (13wks)
$\mathrm{F}$ (5wks)
$\mathrm{F}$ (8wks)
$\mathrm{F}$ (13wks)

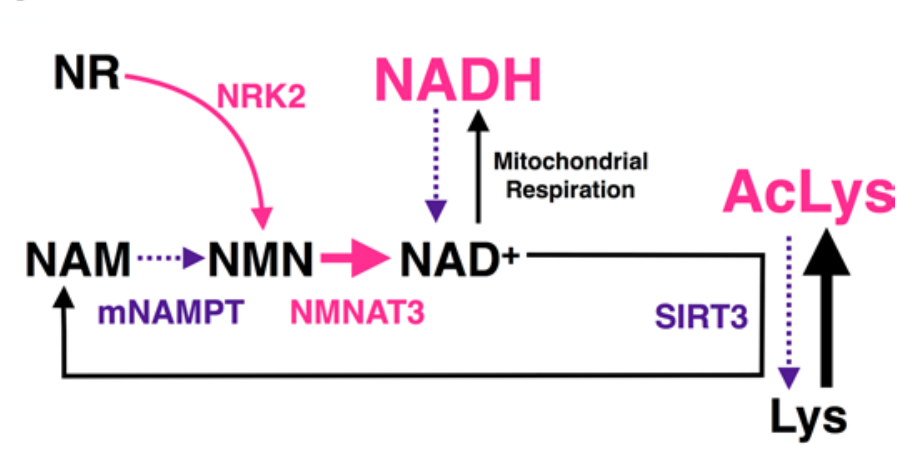

Figure 1. FXN-KO cardiac mitochondria have protein hyperacetylation, redox imbalance, and altered NAD+ homeostasis. (A) Difference in mitochondrial protein acetylation relative occupancy sites (i.e., acetyl peptides quantification corrected for change in protein abundance) between WT and FXN-KO at 5, 8, and 13 weeks of age. Black represents statistically significant $\left(P_{\text {adiusted }} \leq 0.05\right)$ acetyl sites with FCs above 2- or below -2-fold; dark gray represents FCs in between; and light gray represents acetyl sites with no statistically significant FC. (B) Proteomic measurements of OXPHOS complex I subunit levels in WT and FXN-KO heart mitochondria at 5, 8, and 13 weeks of age. Bar line represents sample mean. Subunits shown are statistically significant from respective WT (at 8 and 13 weeks). (C-D) Proteomic measurements of NAD+ biosynthesis proteins NAMPT (C) and NMNAT3 (D) in WT and FXN-KO heart mitochondria at 5, 8, and 13 weeks of age. Bar line represents sample mean. (E) Statistically significant acetyl-Iysine sites in common between the SIRT3-KO whole hearts (green; ^ from ref. 28); and FXN-KO heart mitochondria at 5, 8 , and 13 weeks of age (shades of red). Purple and pink shading represents 1 SD from the mean of the bottom and top, respectively, 25\% SIRT3-KO sites. (F) Schematic summarizing shifts in the mitochondrial NAD+ metabolome in the FXN-KO heart. ${ }^{*} P<0.05$, difference from respective WT values at each age group by Student's $t$ test and Benjamini-Hochberg FDR correction; $n=3$ mice per age group per genotype. W: WT, F: FXN-KO and S: SIRT3-KO. 
A
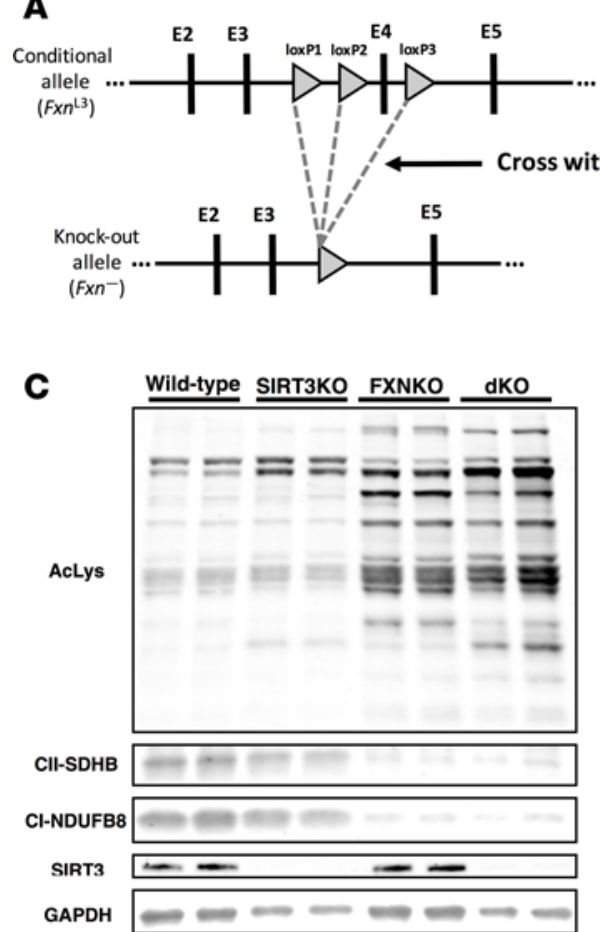

G

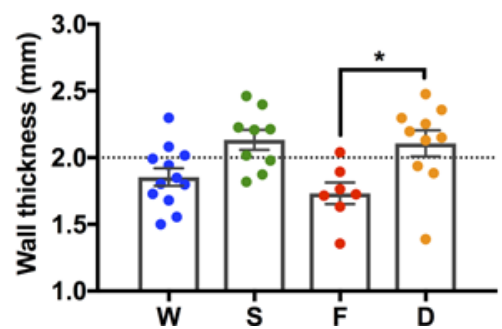

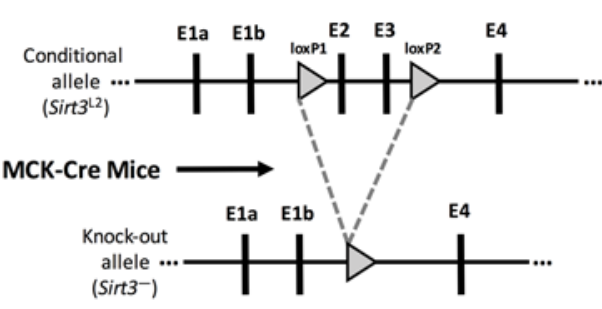

B
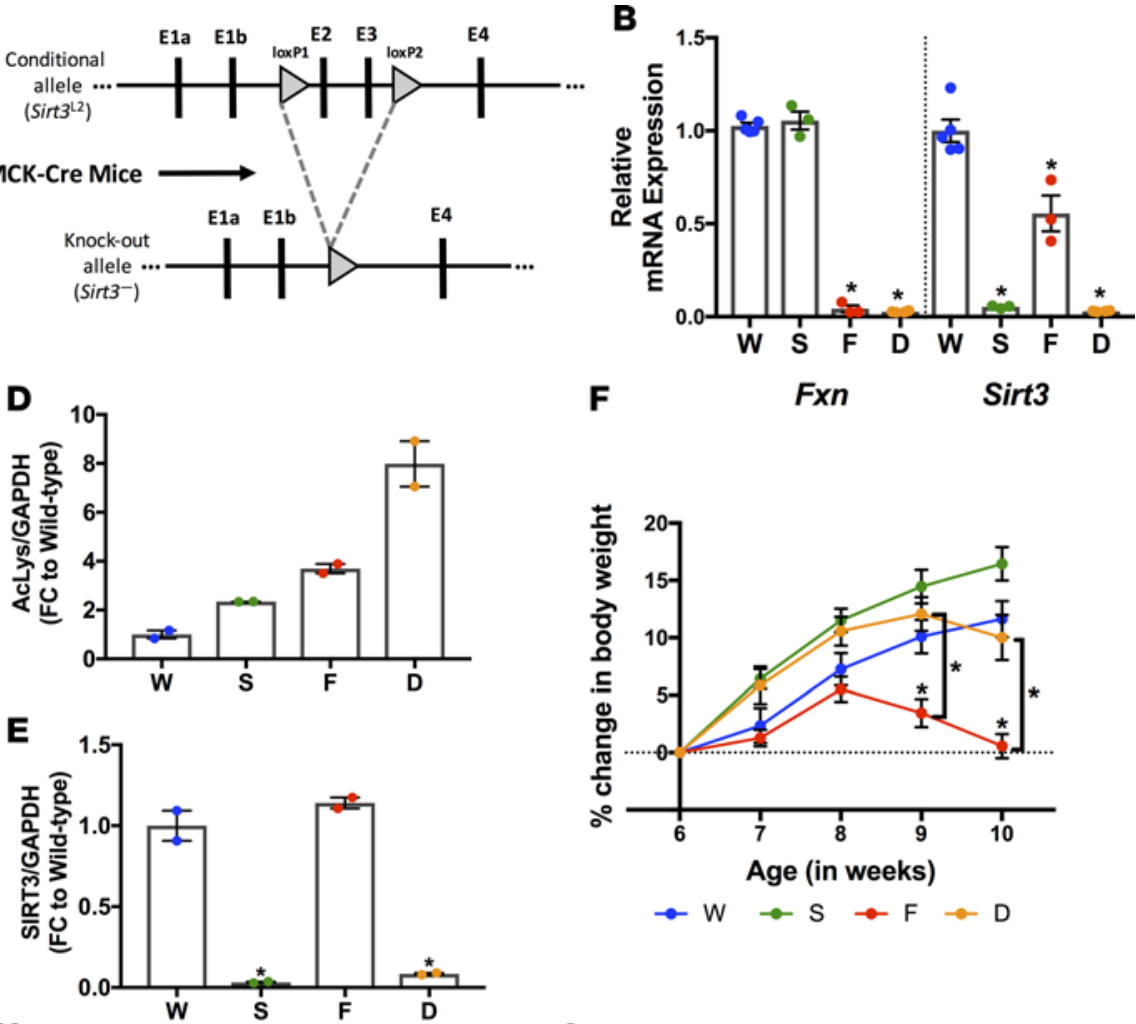

H

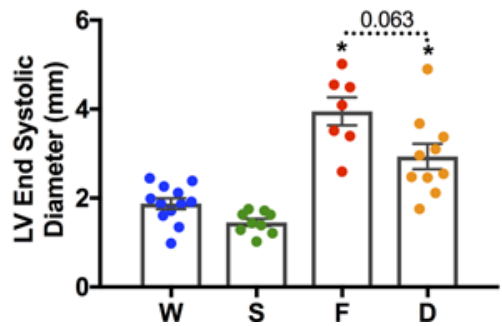

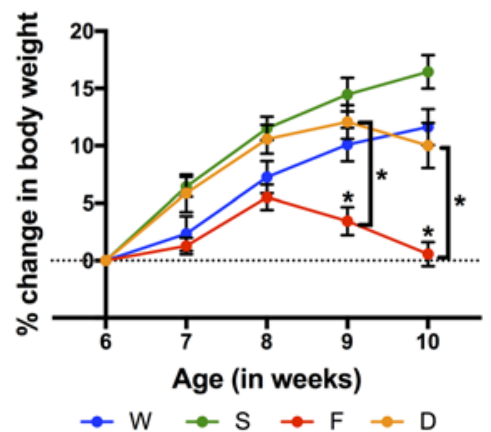

I

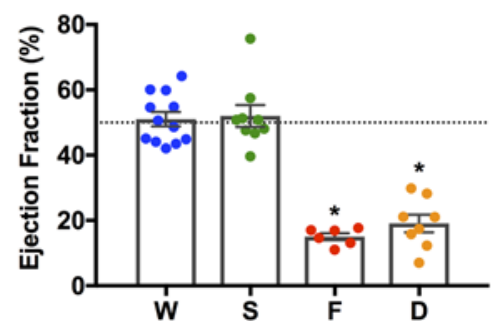

Figure 2. SIRT3 ablation in the FXN-KO heart induces sustained left ventricular wall thickening. (A) Targeting strategy used to generate the cardiac and skeletal muscle-specific Sirt3-KO and Sirt3/Fxn-KO (dKO) mice. A map of the Fxn (left) and Sirt3 (right) genomic locus, which shows the conditional allele (upper panels) and the KO allele (lower panels). Arrowheads indicate LoxP sites. Vertical bars depict respective exons. (B) Cardiac Fxn and Sirt3 mRNA expression. Values are means \pm SEM ( $n=3-5$ mice/group). (C) Cardiac protein acetylation, complex I (NDUFB8) and II (SDHB) subunits, SIRT3, and GAPDH protein expression assessed via Western blotting. (D and E) Western blot quantification of acetylation (D) and SIRT3 expression (E); values are means \pm SEM. (F) Body weight from 6 weeks to 10 weeks of age. Values are means \pm SEM ( $n=11-18$ mice/group). (C) Wall thickness measured via echocardiography at 9-10 weeks of age. Values are means \pm SEM ( $n=7-12$ mice/group). (H) Left ventricle end-systolic diameter measured via echocardiography at $9-10$ weeks of age. Values are means \pm SEM ( $n=7-12$ mice/group). (I) Hemodynamic measure ejection fraction assessed by PV-loop analysis. Values are means $\pm \operatorname{SEM}\left(n=5-12\right.$ mice/group). ${ }^{*} P<0.05$, difference from saline-treated WT as determined by two-way ANOVA for 8 groups (4 genotypes, 2 treatment) and Bonferroni correction. W, WT; F, FXN-KO; S, SIRT3-KO; and D, dKO.

in several genes that regulate the $\mathrm{NAD}^{+}$metabolome ( $\mathrm{NAD}^{+}$precursors and intermediates), consistent with enhanced $\mathrm{NAD}^{+}$synthesis (Supplemental Figure 1E and Supplemental Table 2, gene expression omnibus [GEO], GSE31208). Specifically, we observed increased transcript levels of the muscle-specific nicotinamide riboside kinase 2 (Nmrk2) and decreased transcript levels of nicotinamide phosphoribosyltransferase (Nampt) in FXN-KO hearts. Increased Nmrk2 suggests an enhanced capacity of the FXN-KO heart to take up exogenous $\mathrm{NAD}^{+}$precursors NR and NMN (27), and decreased Nampt suggests reduced de novo synthesis of $\mathrm{NAD}^{+}$in the FXN-KO heart. These observations were validated at the protein level by analysis of the FXN-KO cardiac mitochondrial proteome, which revealed a decrease in the mitochondrial levels of NAMPT and increased expression in the mitochondrial isoform of nicotinamide nucleotide adenylyltransferase 3 (NMNAT3), a protein involved in NMN salvage (Figure 1, C and D, respectively). Altogether, these findings are consistent with increased mitochondrial salvage of $\mathrm{NAD}^{+}$, which suggests increased demand for mitochondrial $\mathrm{NAD}^{+}$in the FXN-KO heart, possibly to maintain $\mathrm{NAD}^{+}$homeostasis. 


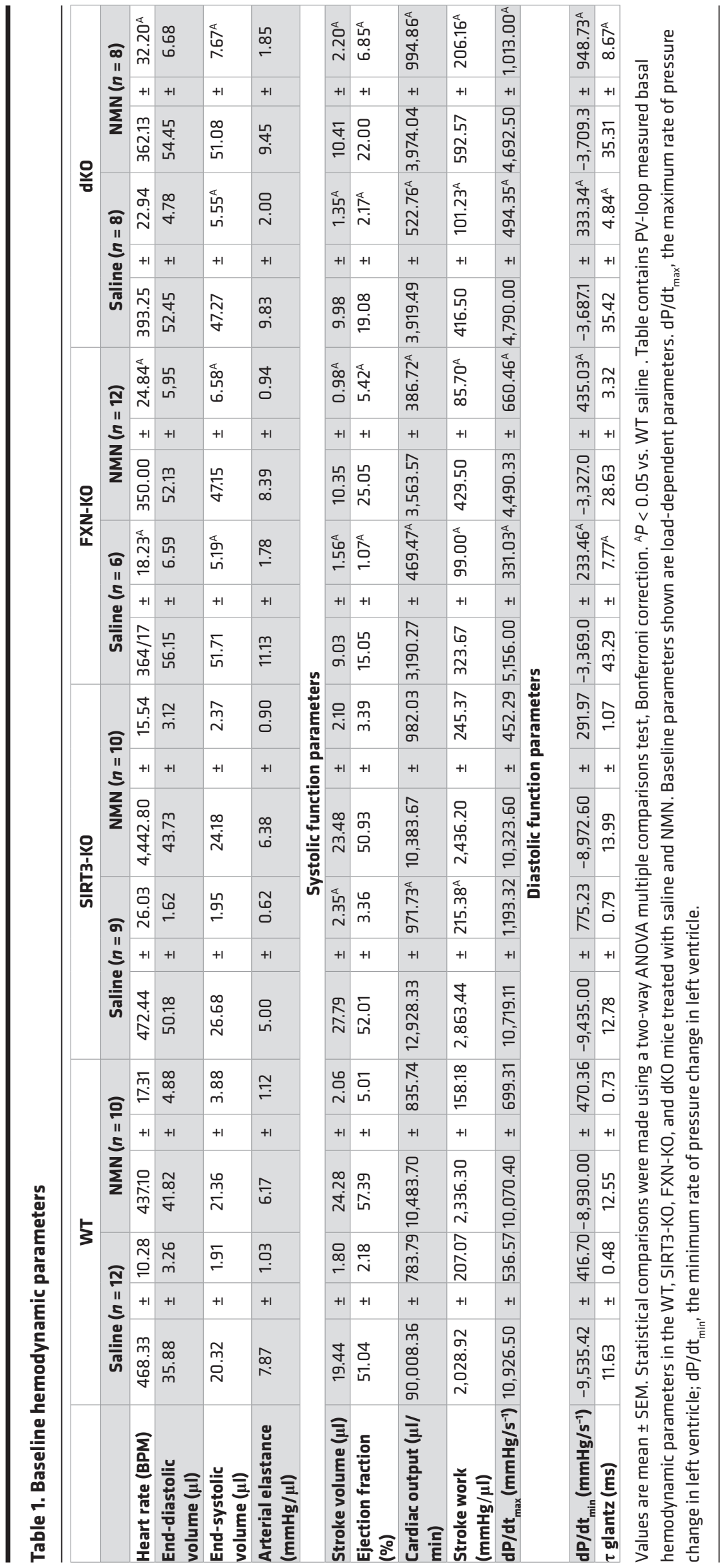

Consequently, decreased mitochondrial $\mathrm{NAD}^{+}$levels in the FXN-KO heart could negatively impact $\mathrm{NAD}^{+}$-dependent SIRT3 deacetylase activity, contributing to the progressive hyperacetylation of mitochondrial proteins in this model (15). Our analyses of gene microarrays showed a progressive decrease in Sirt3 expression - but not in the other mitochondrial sirtuins Sirt4 or Sirt5 - which could further contribute to reduced SIRT3 activity in the FXN-KO heart (Supplemental Figure 1F; validated via qPCR in Figure 2B). Because no tools directly assess SIRT3 activity in vivo, we assessed the acetylation status of cardiac SIRT3 targets as a surrogate for SIRT3 activity. First, we used a previously published multitissue acetyl-proteomic profiling of SIRT3-KO mice as a resource for identifying strong cardiac candidates of regulation by SIRT3 deacetylase activity (28). While both SIRT3-KO and FXN-KO datasets use comparable proteomic technology (isobaric tag-based quantitative proteomics with HCD fragmentation and Orbitrap mass analysis), subtle differences in sample processing and mass spectrometric analyses likely influence slight variation in dynamic range compression (i.e., measured fold change range) (29). Therefore, we used these data only to identify the profile of acetylation changes in the SIRT3-KO and to assess whether similar acetylation patterns occur in the FXN-KO model, but not to directly compare the magnitude of acetylation across these two models.

A list of overlapping acetyl-lysine sites between the SIRT3-KO cardiac acetylome and the FXN-KO cardiac mitochondrial acetylome (i.e., at 5, 8, and 13 weeks of age) revealed 107 sites in common (Supplemental Figure 1G, where acetylation $\log _{2} \mathrm{FC}$ at specific sites in the SIRT3KO heart is rank-ordered [ascending] along the $x$ axis). Next, we plotted $\log _{2} \mathrm{FC}$ of the same sites in the FXN-KO heart at 5, 8, and 13 weeks of age. Acetyl-lysine sites with the highest FCs in the SIRT3-KO heart represent strong candidate targets of SIRT3 deacetylase activity (Supplemental Figure 1G, top 25\%). Additionally, they also represent the most reactive sites in nonenzymatic acetylation reactions in the mitochondria (30). This analysis revealed a 64-fold mean arithmetic difference between the mean of the bottom $25 \%\left(\log _{2} \mathrm{FC}\right.$ of -1$)$ and top $25 \%\left(\log _{2} \mathrm{FC}\right.$ of 5$)$ of SIRT3-KO cardiac acetyl peptides. The large mean difference between the least acetylated and most acetylated peptides in the SIRT3-KO heart illustrates hyperacetylation of strong candidate 
SIRT3 sites (i.e., top 25\%) with loss of SIRT3, and it confirms the high reactivity of these sites to acetylation. By contrast, in the FXN-KO, a mean arithmetic difference of -1.4-fold at 5 weeks, 1.2-fold at 8 weeks, and 1.0-fold at 13 weeks was observed. The negative and small mean arithmetic differences indicate low acetylation occurring at strong candidate SIRT3 sites (i.e., top 25\%) in the FXN-KO heart with disease progression, which suggests active SIRT3 deacetylase regulation at these sites.

Next, we evaluated the shifts in acetylation in the FXN-KO acetyl-proteomic dataset for strong candidate SIRT3 sites (i.e., top 25\%) and acetylation sites unlikely to be targeted by SIRT3 (i.e., bottom 25\%). Linear regression modeling revealed no correlation between the bottom $25 \%$ of SIRT3-KO sites and the corresponding FXN-KO sites at 8 and 13 weeks of age (Supplemental Figure 1H), suggesting aberrant acetylation occurring at these sites in both models, sites not likely regulated by SIRT3 deacetylase activity. In contrast, a strong, significant positive correlation was observed between the top 25\% of SIRT3-KO sites and the corresponding FXN-KO sites at 8 weeks, but it was lost at 13 weeks (Supplemental Figure 1I). These data suggest functional SIRT3 deacetylase activity in the late-stage FXN-KO heart.

Lastly, we monitored changes in the mean $\log _{2} \mathrm{FC}$ of these 107 overlapping sites of acetylation in the FXN-KO (Figure 1E). The rank-ordered SIRT3-KO sites binned into quartiles showed increasing mean $\log _{2} \mathrm{FC}$ of acetylation, where the top $25 \%$ represent strong candidate SIRT3 target sites. The corresponding FXN-KO datasets did not show the same increase in the mean $\log _{2} \mathrm{FC}$ from the bottom $25 \%$ to the top $25 \%$, supporting active SIRT3 deacetylase activity at these reactive, strong candidate SIRT3 sites. Notably, the $\log _{2} \mathrm{FC}$ at these strong candidate SIRT3 sites in the FXN-KO datasets still demonstrated statistically significant hyperacetylation, as compared with WT controls (i.e., 8 weeks, $\log _{2}$ FC $1.77 \pm 0.25$ SEM, and 13 weeks, $\log _{2}$ FC $1.34 \pm 0.20$ SEM). Taken together, these data suggest reduced and/or insufficient SIRT3 deacetylase activity in the FXN-KO heart to combat the increasing protein acetylation. These data depict a system of mitochondrial dysfunction, whereby redox imbalance in the FXN-KO heart may contribute to mitochondrial protein hyperacetylation, altered $\mathrm{NAD}^{+}$homeostasis, and impaired SIRT3 activity (Figure $1 F)$. Furthermore, these data suggest $\mathrm{NAD}^{+}$therapy with $\mathrm{NMN}$ supplementation could be a strategy to activate SIRT3 and/or restore redox imbalances in the FXN-KO heart.

SIRT3 ablation in the FXN-KO heart induces sustained left ventricular wall thickening. Thus, we set out to test NMN precursor supplementation as a therapeutic means to augment SIRT3 activity in the FXN-KO heart. We hypothesized that NMN-induced mitochondrial $\mathrm{NAD}^{+}$production and enhanced SIRT3 activity would alleviate repressive acetylation marks to improve cardiac metabolism and function. To directly test this hypothesis, we generated SIRT3-KO and SIRT3-KO/FXN-KO double KO (dKO) mouse models, under the control of the muscle creatine kinase (MCK) Cre-driven promoter (i.e., cardiac/skeletal muscle tissue-specific, Figure 2A), as was previously used to generate the FXN-KO mouse (16). These models enabled us to assess the direct role of SIRT3 in mediating any effects of NMN in the FXN-KO heart.

First, we characterized these new models and assessed the basal role of SIRT3 in the FXN-KO heart. The FXN-KO had effective ablation of Fxn at the mRNA level (Figure 2B) and reduced complex I (NDUFS1-5,7,8), complex II (SDHA-C), and complex III (Rieske) subunit protein levels (Figure 1B and Supplemental Figures 1, A-D), established readouts of FXN ablation (17). The SIRT3-KO model had effective ablation of Sirt3 at the mRNA (Figure 2B) and protein levels in the heart (Figure 2, C and E). Importantly, the dKO mouse model showed both ablation of FXN and SIRT3 (Figure 2, B, C, and E). As observed in the hearts of global SIRT3-KO mice $(13,31)$, deletion of Sirt3 resulted in mitochondrial protein hyperacetylation, which was exacerbated in the dKO heart compared with the FXN-KO heart (Figure 2, C and D). Hyperacetylation in the $\mathrm{dKO}$ further supports a functional but insufficient SIRT3 activity in FXNKO mouse hearts. Interestingly, a lack of Sirt3 in these models contributed to weight gain in the SIRT3$\mathrm{KO}$ and dKO models, as compared with saline-treated WT mice (Figure 2F). This weight gain was not observed basally in global (13) or in skeletal muscle- or liver-specific SIRT3-KO models (32). Both the dKO and FXN-KO lost weight at the end-stage of disease (Figure 2F), consistent with cardiac cachexia (16).

Morphologically, the SIRT3-KO exhibited spontaneous hypertrophy, as determined by increased left ventricular (LV) wall thickness and decreased LV end-systolic diameter (LVESD) measured by echocardiography (Figure 2, G and H, respectively). These data depicting cardiac hypertrophy in the SIRT3-KO as compared with WT controls are not statistically significant, as these differences are overshadowed by the severe hypotrophy of the FXN-KO HF models. However, outside of the context of the FXN-KO and dKO models, these morphological differences between WT and SIRT3-KO mice are statistically significant (Student's $t$ test, $P \leq$ 0.05). Furthermore, these data are congruent with the literature, which describes spontaneous and age-induced 
cardiac hypertrophy in the global SIRT3-KO $(9,10)$. FXN-KO mice, like FRDA patients (33), develop LV hypertrophy that leads to an enlarged, dilated LV end-stage disease. Interestingly, Sirt3 ablation in the dKO resulted in sustained LV wall thickening, as shown by a statistically significant increase in LV wall thickness and near significant decrease in LVESD, as compared with the FXN-KO (Figure 2, G and H, respectively).

Grossly, the FXN-KO and dKO mice appeared no different over the course of disease progression (data not shown). FXN-KO is a model of severe dilated cardiomyopathy, with hearts burdened by large ventricular load at end-stage disease (33). The enlarged LV and thinned LV wall contribute to increased preload (e.g., as measured by end-diastolic volume [EDV] and end-systolic volume [ESV]; Table 1). Increased preload in the FXN-KO hearts depresses the relationship between volume ejected with each stroke and the volume remaining within the LV with each cardiac cycle, which is reflected in load-dependent functional parameters whose lower values are indicative of worsened cardiac function. Specifically, the FXN-KO have poorer cardiac function compared with WT controls, as measured by the load-dependent parameter ejection fraction (EF), measured by pressure-volume loop (PV-loop) analysis (Figure 2I). The dKO heart showed comparable EF to the FXN-KO (Figure 2I). Taken together, cardiac Sirt3 ablation drives LV hypertrophy, with no functional consequence in the SIRT3-KO and $\mathrm{dKO}$ at baseline, when compared with the WT or FXN-KO models. Indeed, previous studies have described global SIRT3-KO mice as grossly comparable with WT controls $(10,12,13)$.

$N M N$ in the FXN-KO improves diastolic function and normalizes systolic function in a SIRT3-dependent manner. To test whether NMN supplementation enhances $\mathrm{NAD}^{+}$production and improves cardiac function in FXN-KO mice, and to determine the role of SIRT3 in mediating the beneficial effects of NMN supplementation, we treated WT, SIRT3-KO, FXN-KO, and dKO mice with NMN (500 mg/kg) 2 times per week for 6 weeks (Figure 3A). First, to assess the efficacy of this treatment paradigm, we measured cardiac $\mathrm{NAD}^{+}$, as well as several NAD ${ }^{+}$-related metabolites. We found that chronic NMN supplementation resulted in a statistically significant increase in $\mathrm{NAD}^{+}$levels in the FXN-KO and $\mathrm{dKO}$ models, as compared with saline-treated WT mice (Figure 3B). Interestingly, quantitative mass spectrometric analysis of tissue metabolites also revealed an increase in NMN levels basally and with NMN supplementation, as compared with saline-treated WT controls (Supplemental Figure 2A). These data are consistent with the enhanced scavenging of NMN in the FXN-KO heart, as described above (Figure 1). To assess whether NMN-induced $\mathrm{NAD}^{+}$production could enhance SIRT3 deacetylase activity, we evaluated global mitochondrial acetylation via Western blot analysis. dKO hearts display 4 times more global mitochondrial acetylation than the SIRT3-KO, and 2 times more than the FXN-KO, in the heart (Figure 2, C and D). However, NMN supplementation did not alter global mitochondrial protein hyperacetylation in these models (Supplemental Figure 2, B and C), suggesting that specific targets of SIRT3 mediate the effects of NMN in the FXN-KO, as opposed to global shifts in protein acetylation.

Overall, NMN treatment did not affect end-stage weight loss in the FXN-KO mice but it decreased overall weight gain throughout the study in the $\mathrm{dKO}$ model when compared with saline-treated $\mathrm{dKO}$ mice (Supplemental Figure 2, D and E). Next, we measured cardiac morphology and function using echocardiography followed by terminal PV-loop analysis. SIRT3-KO and dKO mice treated with NMN, as compared with their saline-treated counterparts, maintained a thickened LV wall of about $2 \mathrm{~mm}$ on average (Figure $3 \mathrm{C}$ and Figure 2G, respectively). By contrast, NMN supplementation in the FXN-KO attenuated thinning of the $\mathrm{LV}$ wall with ventricular dilation (Figure $3 \mathrm{C}$ vs. Figure $2 \mathrm{G}$ ). Echocardiographic analyses comparing FXN-KO mice at 7 weeks (1-2 weeks of NMN treatment) and 9 weeks (3-4 weeks of NMN treatment) reveal attenuated development of LV wall thinning (Supplemental Figure 2F). Despite a thicker LV wall, FXN-KO hearts remained dilated, as load-dependent LVESD was unchanged with NMN treatment (Supplemental Figure $2 \mathrm{G}$ ), consistent with the substantial preload in this model.

Next, invasive hemodynamic measurements were performed to assess the impact of NMN on systolic and diastolic parameters (Tables 1 and 2). Load-dependent parameters, such as EF (Figure 3D), remained depressed in the FXN-KO and dKO models, compared with WT controls (Table 1). In contrast, NMN administration in the FXN-KO had substantial effects on load-independent measures of ventricular contractility and compliance when measured by PV-loop analyses (Table 2). NMN treatment reduced both $\tau$ and the slope of the end diastolic pressure volume relationship (EDPVR), corresponding to improved diastolic filling time and stiffness (Figure 3, E and F, respectively). At baseline, saline-treated FXN-KO mice had reduced contractility, as measured by end systolic pressure volume relation (ESPVR), maximal change in pressure over time $\left(\mathrm{dP} / \mathrm{dt}_{\max }\right)$ vs. EDV, and maximal elastance $\left(\mathrm{E}_{\max }\right)$; remarkably, these three measures 
A

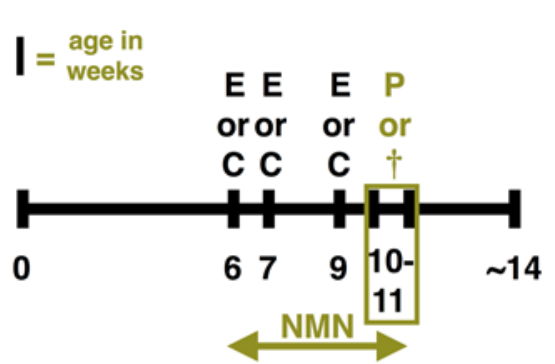

D

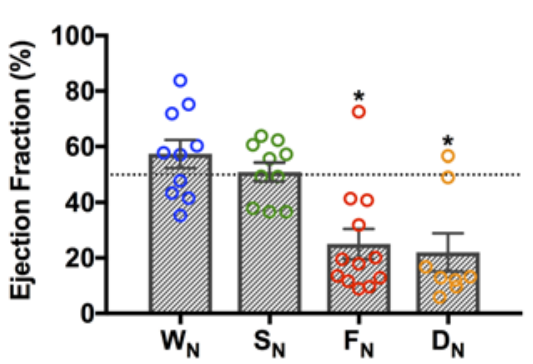

G

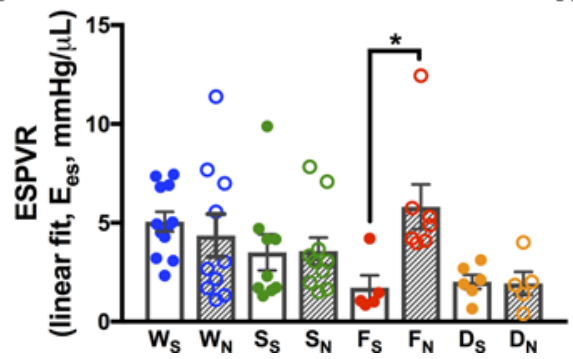

B

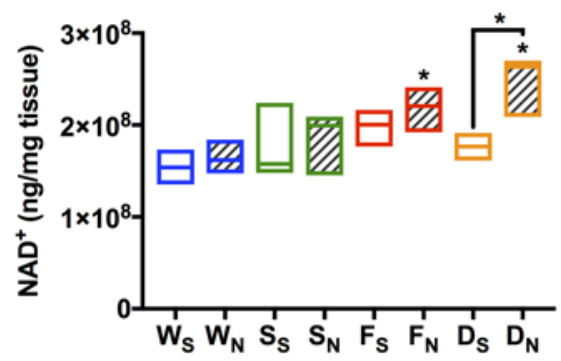

E

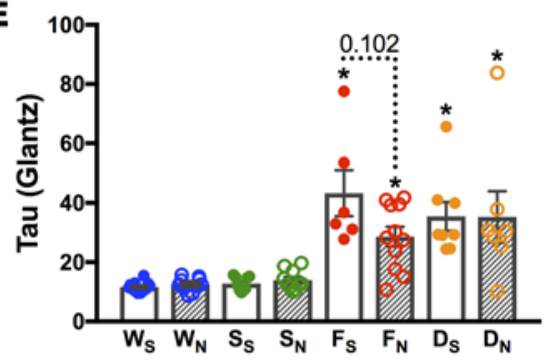

H

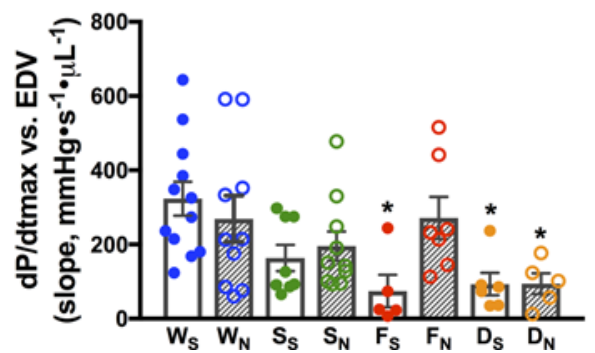

C

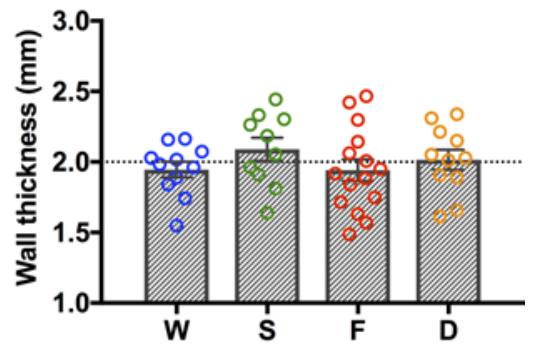

Figure 3. NMN in the FXN-KO improves diastolic and normalizes systolic function in a SIRT3-dependent manner. (A) Experimental schedule of treatment and clinical phenotyping protocols. E, echocardiography; C, noninvasive monitoring by CLAMS; P, PV-loop analysis; and †, sacrifice and collection of tissues for further analysis. Terminal procedures represented by olive-colored box. (B) Metabolomics profiling of cardiac NAD+ levels. Values are means \pm SEM ( $n=3-5$ mice/group). (C) Wall thickness in NMN-treated animals measured via echocardiography at 9-10 weeks of age. Values are means \pm SEM ( $n$ = 7-12 mice/group). (D-F) Hemodynamic measures assessed by PV-loop analysis: ejection fraction (D), active relaxation ( $\tau$, E), passive filling (linear end

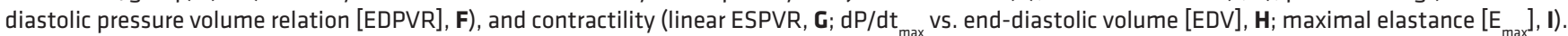
Values are means \pm SEM (E and $\mathbf{G}-\mathbf{I}, n=5-10$ mice/group; $\mathbf{D}$ and $\mathbf{F}, n=7-10$ mice/group). Hashed line at $50 \%$ represents normal mouse ejection fraction. ${ }^{*} P$ $<0.05$, difference from saline-treated WT as determined by two-way ANOVA for 8 groups (4 genotypes, 2 treatment) and Bonferroni correction. W, WT; F, FXN-KO; S, SIRT3-KO; D, dKO; subscript S, saline; and subscript N, nicotinamide mononucleotide (NMN).

of contractility were restored with NMN treatment (Figure 3, G-I). These striking NMN-induced improvements in FXN-KO mice were not dependent on NMN's effects on peripheral vascular resistance as measured by arterial elastance (Table 1). Together, these data show that NMN supplementation in the FXN-KO restored cardiac contractility and compliance via cardiac intrinsic effects of NMN.

The dKO also displayed comparable severity in cardiac diastolic and systolic dysfunction to the FXN-KO mice (Figure 3, E-I). Notably, the SIRT3-KO mice displayed a strong trend in contractile dysfunction (Figure 3, G-I), while diastolic function was similar to saline-treated WT mice (Figure 3, E and F). Despite impairments in contractility, the SIRT3-KO mice had comparable cardiac performance to WT mice, as measured by EF (Figure 2I and Figure 3D). The restoration of cardiac compliance and contractility in the NMN-treated FXN-KO mice required SIRT3, as NMN could not rescue cardiac function in the SIRT3-KO and dKO hearts (Figure 3, E-I). Furthermore, these SIRT3-dependent effects of NMN are cardiac intrinsic, as the saline- and NMN-treated SIRT3-KO and dKO models showed no difference in arterial peripheral resistance compared with saline-treated WT controls (data not shown). Altogether, these findings reflect SIRT3-dependent improvements in contractility, active relaxation, and passive filling in the FXN-KO mouse heart. 


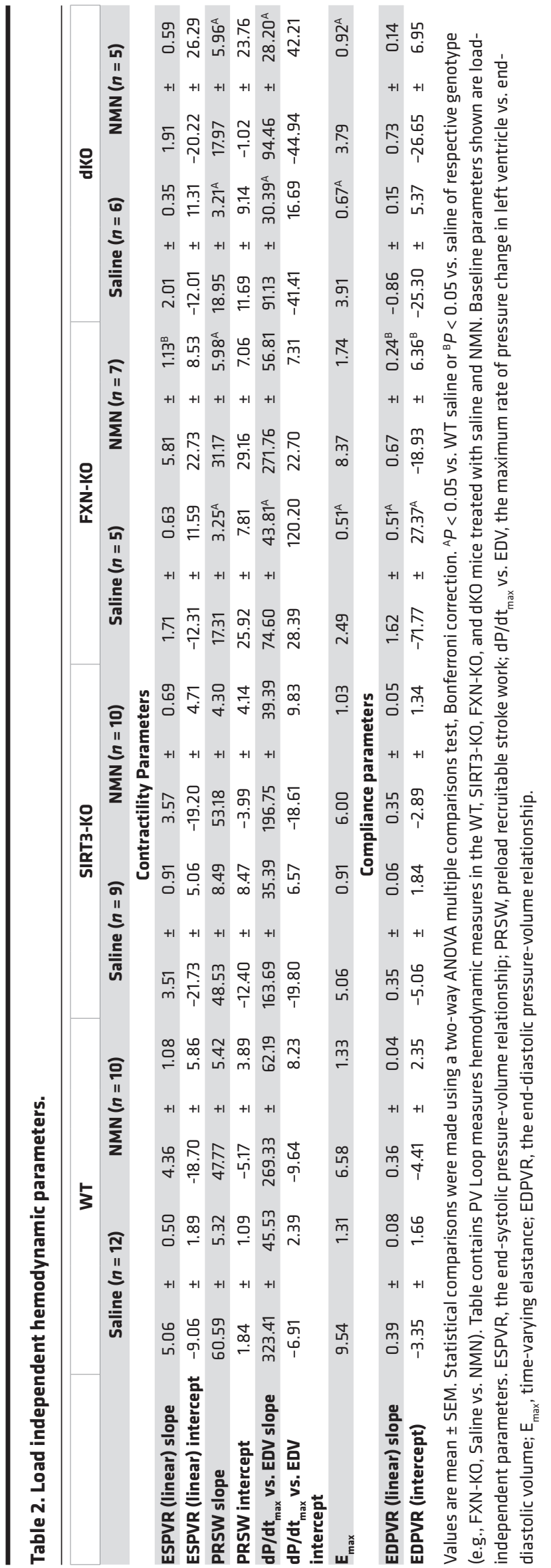

NMN improves cardiac energy generation and utilization in a SIRT3dependent manner. The restoration of contractile function and improvements in ATP-dependent active relaxation in the FXN-KO mice suggested NMN treatment led to positive alterations in cardiac bioenergetics. As such, we comprehensively profiled energy metabolites using mass spectrometry-based metabolomics and identified changes in high-energy phosphate-bearing metabolites upon NMN treatment. Both the $\mathrm{PCr} / \mathrm{ATP}$ and $\mathrm{PCr} / \mathrm{Cr}$ ratios in the FXN-KO trended toward increased upon NMN supplementation (Figure 4, A and B, respectively), which appeared to be SIRT3 dependent; interestingly, dKO mice displayed elevated ratios that trended toward a reduction upon NMN treatment. Further characterization of possible energy shifts upon NMN treatment revealed no change in the ATP/AMP ratio and no difference in AMPK phosphorylation status (Supplemental Figure 3, A-D). These data suggest that the increased high-energy phosphate-bearing metabolite ratios reflect shifts in energy utilization, rather than overall ATP generation, in the FXN-KO heart.

Thus, we next analyzed cardiac efficiency (CE) (i.e., the ratio of energy used for mechanical work to overall energy available) in the PV-loop data collected from saline-treated FXN-KO and dKO failing hearts and found significantly lower CE (Figure 4C). Depressed CE is a salient feature of ventricular energetics during HF (34-36). Strikingly, CE in the FXN-KO was normalized with NMN supplementation (Figure 4C). Restoration of CE required SIRT3, as CE remained depressed in $\mathrm{NMN}$-treated $\mathrm{dKO}$ hearts. An additional parameter of energy efficiency in the heart is the ventriculoarterial coupling (VC) ratio, which measures the efficiency of the heart to transfer mechanical energy (ME) from the ventricle to hydraulic energy in the aorta during contraction (34-36). In failing hearts, decreased systolic contractility (Figure 3, G-I) contributes to decreased ventricular systolic elastance, which corresponds to an increase in the VC ratio at baseline in the saline-treated FXN-KO and $\mathrm{dKO}$ heart failing models (Figure 4D). Ultimately, this indicates that a greater fraction of work done by the ventricle is wasted as potential energy (PE) rather than as $\mathrm{ME}$ that enables cardiac work in these models. Remarkably, NMN treatment resulted in a complete normalization of the VC ratio in the FXN-KO mice, which again was an improvement achieved in a SIRT3-dependent manner (Figure 4D).

Next, we used PV-loop analyses to measure the total cardiac available energy for each cardiac cycle (pressure-volume area, PVA). PVA is the sum of ME (i.e., cardiac/stroke work) and PE (i.e., basal metabolism or wasted) (34). Energy pie charts depict diminished PVA at baseline in the saline-treated FXN-KO and dKO mice (Figure 4E; also see Supplemental Figure 3E). Furthermore, a greater proportion of PVA was spent as $\mathrm{PE}$, rather than $\mathrm{ME}$, at baseline the FXN-KO and dKO hearts (Figure 4, E and F, PE in dark brown and $\mathrm{ME}$ in olive green). Quantification of the FXN-KO and dKO heart failing models reveal greater wasted energy, $\mathrm{PE}$, as compared to the saline-treated WT controls (Figure 4F). Strikingly, NMN supplementation normalized the contributions of PE and ME to PVA in the FXN-KO heart but not in the dKO heart. These NMN-induced improvements in myocardial energetic utilization were achieved via a SIRT3-dependent decrease in PE, which demonstrates that the decreased energy wasting with NMN in the FXN-KO heart requires 
A

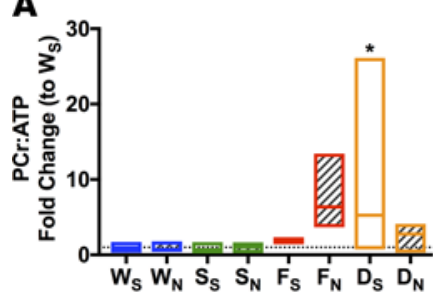

B

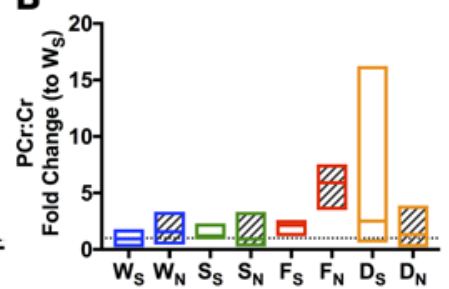

C

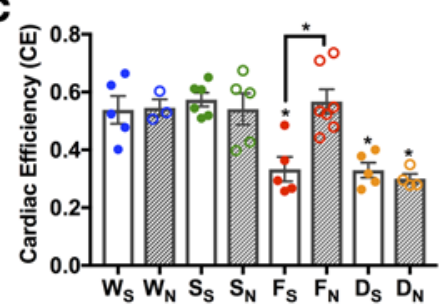

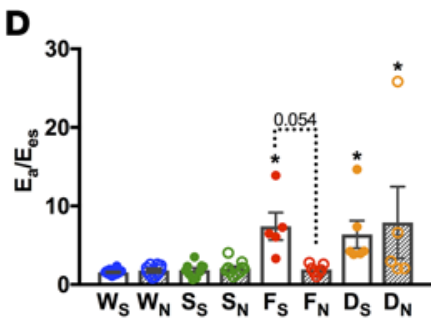

$\mathbf{E}$

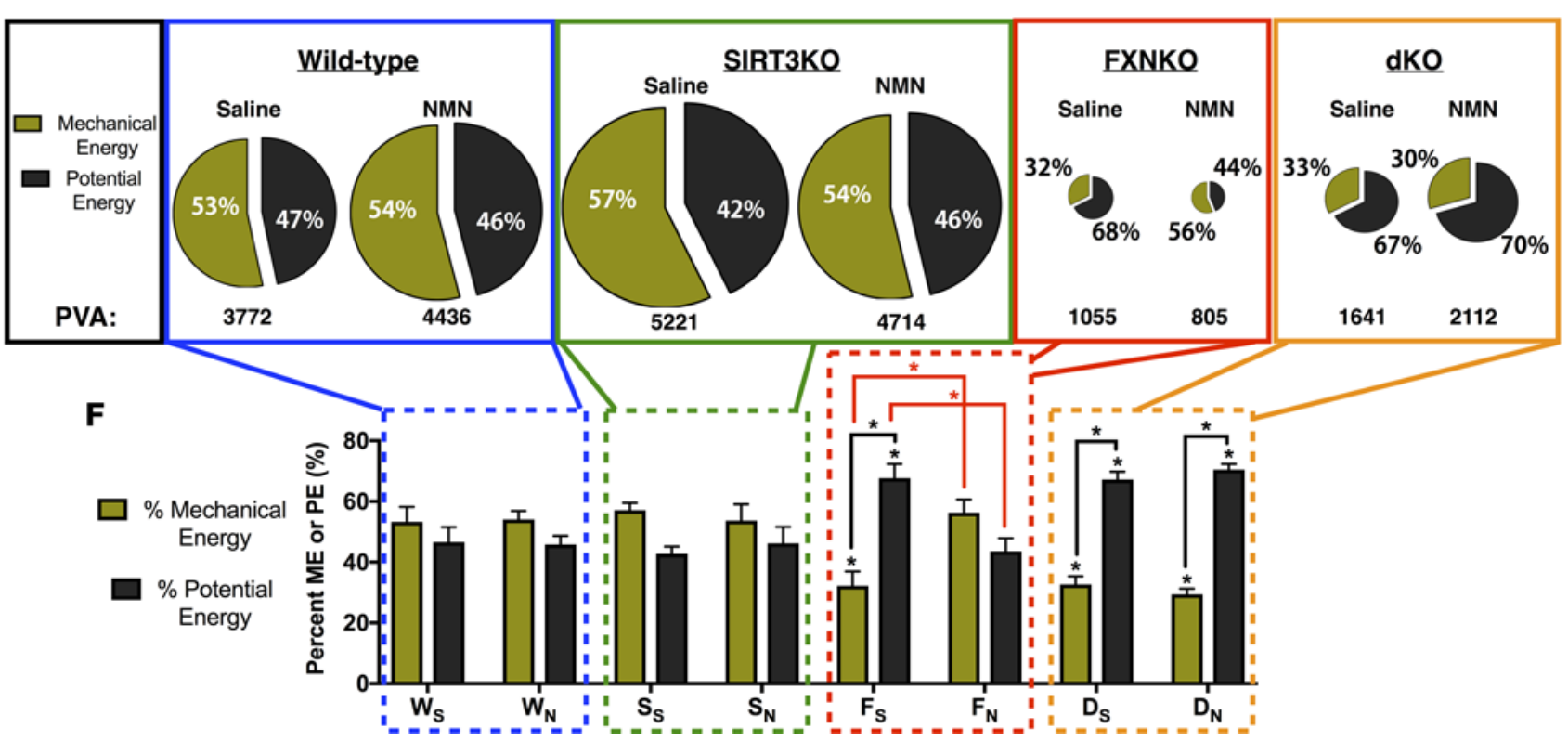

Figure 4. NMN reduces energy wasting and improves energy utilization in the FXN-KO heart. (A and $B$ ) High-energy phosphate-bearing metabolite ratios, $\mathrm{PCr} / \mathrm{Cr}$ (A) and PCr/ATP (B). Bar line represents sample mean ( $n=3-5$ mice/group). (C) Cardiac efficiency and (D) ventriculoarterial energy transfer ratio. $E_{a}$, effective arterial elastance; $E_{e s}$, ventricular end-systolic elastance. Values are means \pm SEM ( $n=5-12$ mice/group). (E) Energy contribution diagrams ( $n=5-12$ mice/group). Mechanical energy (olive green) and potential energy (dark brown) contribute to overall available energy (PVA). (F) Quantification of the energy contribution diagrams in $\mathbf{E}$. ${ }^{*} P<0.05$, difference from saline-treated WT as determined by two-way ANOVA for 8 groups ( 4 genotypes, 2 treatment) and Bonferroni correction. W, WT; F, FXN-KO; S, SIRT3-KO; D, dKO; subscript S, saline; and subscript N, NMN.

cardiac SIRT3 (Supplemental Figure 3F). Altogether, NMN improves cardiac energy utilization and efficiency in a SIRT3-dependent manner.

$N M N$ reduces whole-body energy expenditure in a SIRT3-dependent manner. In addition to shifts in ventricular energetics, $\mathrm{HF}$ is associated with changes in whole-body energy balance $(37,38)$. To assess the impact of chronic NMN supplementation on whole-body energy metabolism in these mouse models, we comprehensively measured several parameters of respiration using indirect calorimetry, including respiratory exchange (i.e., oxygen consumption, $\mathrm{VO}_{2}$, to carbon dioxide release, $\mathrm{VCO}_{2}$ ), food intake, and activity (39, 40). Energy balance is achieved when energy intake (i.e., parameter: food intake, Supplemental Figure 4A) matches energy expended (i.e. parameters: energy expenditure [EE], Figure 5A; heat expenditure, Supplemental Figure 4B; and locomotive activity Supplemental Figure 4C). In saline-treated animals, the FXNKO heart-failing model appears to maintain energy balance but has an overall decrease in whole-body energy requirements, as shown by a trending decrease in both energy intake (Supplemental Figure 4A) and in energy expended (Figure 5A and Supplemental Figure 4, B and C), compared with WT saline-treated controls. These data are consistent with reduced available energy in the FXN-KO heart (Figure 4E). Surprisingly, the SIRT3-KO mice displayed an energy imbalance. While energy intake was not different compared with WT mice (Supplemental Figure 4A), EE was reduced (Figure 5A and Supplemental Figure $4 \mathrm{~B}$ ). This decrease in energy spent is consistent with the weight gain observed in these mice (Figure $2 \mathrm{~F}$ ), which suggests energy storage in the form of body mass, rather than energy used for whole-body cellular metabolism. This energy imbalance was not observed in global (13) or in skeletal muscle- or liver-specific SIRT3-KO models (32). Because total EE is mostly generated from basal metabolism - the energy spent 
A

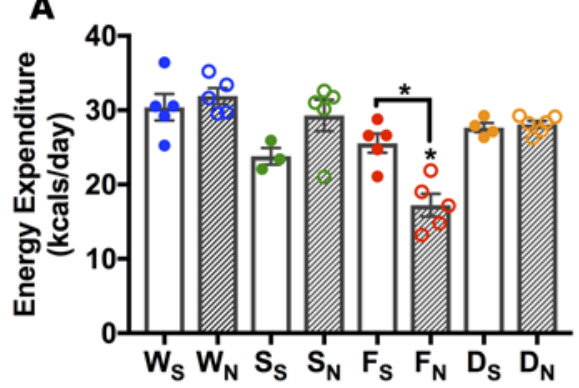

B

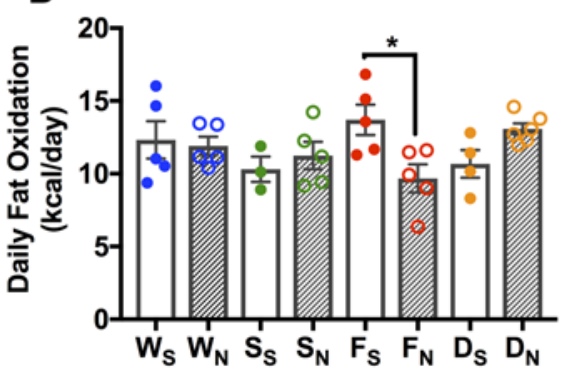

C

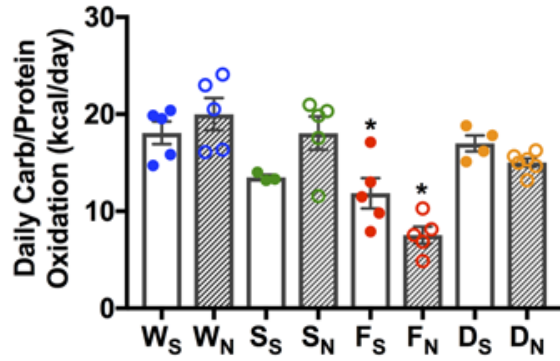

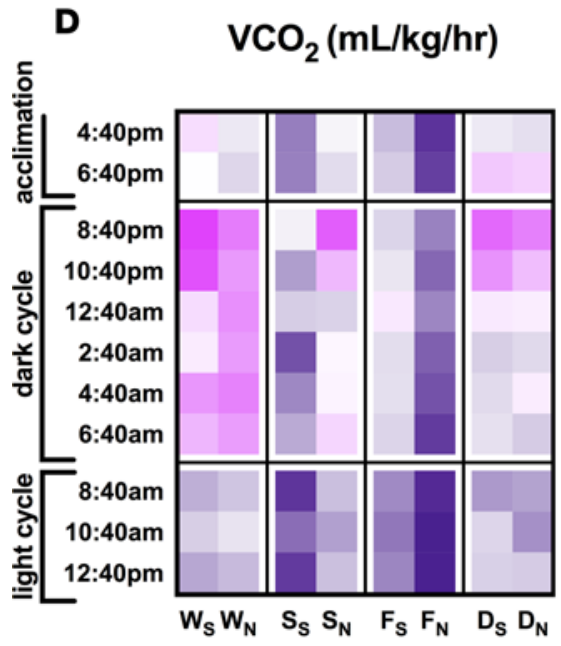

$\mathrm{VO}_{2}(\mathrm{~mL} / \mathrm{kg} / \mathrm{hr})$

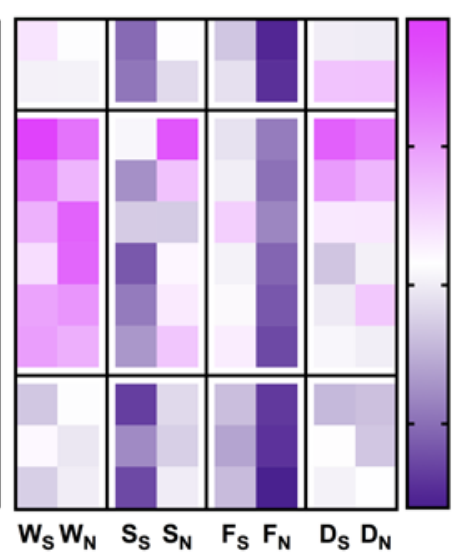

E

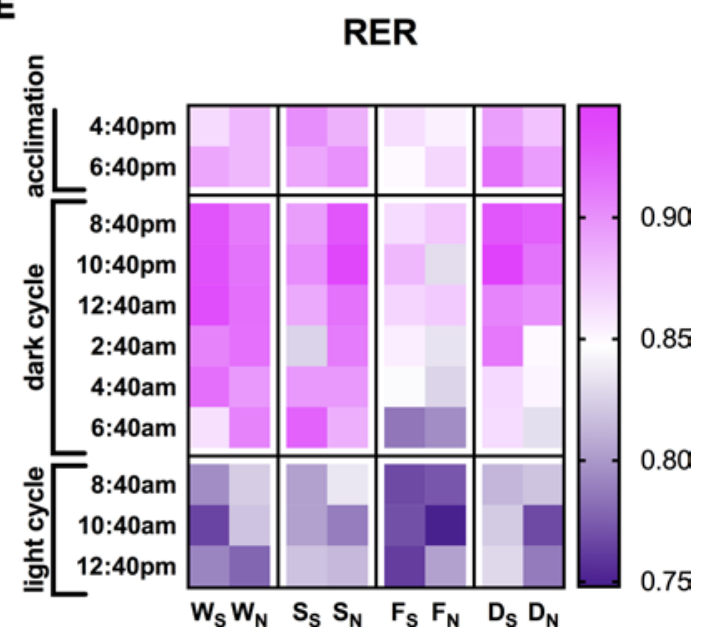

Figure 5. NMN reduces daily energy expenditure in the FXN-KO mouse in a SIRT3-dependent manner. (A) Energy expenditure, (B) fat oxidation, (C) carbohydrate oxidation, (D) oxygen consumption $\left(\mathrm{VO}_{2}\right)$ and carbon dioxide production $\left(\mathrm{VCO}_{2}\right)$, and $(\mathbf{E})$ respiratory exchange ratio (RER). Values are means \pm SEM ( $n=3-6$ mice/group). ${ }^{*} P<0.05$, difference from saline-treated WT as determined by two-way ANOVA for 8 groups (4 genotypes, 2 treatment) and Bonferroni correction. W, WT; F, FXN-KO; S, SIRT3-KO; D, dKO; subscript S, saline; and subscript N, NMN.

to sustain the metabolic activity of cells in all tissues of the body $(39,40)$ - we measured cellular macronutrient oxidation in these models. Reduced carbohydrate oxidation appeared to largely account for the reduced EE in the FXN-KO and in the SIRT3-KO mice (Figure 5C). Macronutrient oxidation reflects cellular respiration, and we found that shifts in respiration appear to be the underlying cause of the reduced $\mathrm{EE}$ in both the FXN-KO and SIRT3-KO mice (Figure 5, D and E). $\mathrm{VO}_{2}$ and $\mathrm{CO}_{2}$ are both greatly depressed in these models, resulting in depressed basal metabolic rate in these models. Interestingly, Sirt3 ablation appears to drive energy imbalance in the dKO heart failing model, as well. But in contrast to the SIRT3-KO model, SIRT3 loss in the dKO increased energy spent in the dKO (Figure 5A and Supplemental Figure 4, $\mathrm{B}$ and C) relative to the energy intake in the failing heart (Supplemental Figure 4A). Consistent with this finding, loss of SIRT3 in the dKO drove an increased respiratory exchange (Figure 5, D and E). Thus, while the FXN-KO mice have an overall reduction in bodily energy requirements (i.e., reduction in both energy intake and energy spent), they appear to maintain energy balance. This whole-body energy balance is lost with ablation of SIRT3 in the dKO model, as well as in the SIRT3-KO model.

Remarkably, NMN supplementation further reduced whole-body daily energy needs in the FXN-KO mice (Figure 5A and Supplemental Figure 4, A and B), similar to the significant reduction in cardiac energy wasting or PE with NMN (Figure 4, E and F). This reduction in EE was achieved by a decrease in daily FA and carbohydrate oxidation rates (Figure 5, B and C), due to reduced respiration (Figure 5, D and E). Consistent with the NMN-induced shifts in cardiac energy utilization resulting in enhanced CE (Figure 4, $\mathrm{C}$ and $\mathrm{F}$ ), NMN-induced lowering in daily energy needs in the FXN-KO mice led to comparable locomotive activity as compared with the saline-treated FXN-KO (Supplemental Figure 4C), suggesting improved whole-body energy utilization in the NMN-treated FXN-KO mice. In contrast, NMN drives increases in EE in the SIRT3-KO mouse (Figure 5A and Supplemental Figure 4B) without altering energy intake (Supplemental Figure $4 \mathrm{~A})$. The increase in EE was reflected in increased daily carbohydrate oxidation rates 
(Figure 5C) due to increased cellular respiration (Figure 5, D and E). The NMN-induced reduction in daily energy requirements in the FXN-KO was lost in the $\mathrm{dKO}$, likely due to augmented energy spending with Sirt3 ablation, as observed in the SIRT3-KO mice (Figure 5 and Supplemental Figure 4). Altogether, these data point to a SIRT3-dependent improvement in whole-body energy utilization upon NMN treatment, which was achieved by a reduction in daily energy needs in the FXN-KO mouse.

NMN reduces cardiac glucose metabolism in a SIRT3-dependent manner. NMN treatment in the FXN-KO mice mediated a reduction in whole-body energy needs, as reflected in a reduction in substrate metabolism. Given the NMN-induced decrease in energy wasting in the FXN-KO heart, these findings prompted us next to evaluate whether this depressed whole-body substrate metabolism manifests within the heart. The FXN-KO mice at baseline have elevated serum lactate (Figure 6A), indicative of elevated systemic glucose metabolism and/or lower mitochondrial pyruvate oxidation. Indeed, we found the FXN-KO heart had hampered pyruvate oxidative rates (Figure 6B), supporting defective mitochondrial metabolism. To further interrogate glucose metabolism, we monitored the abundance of glycolytic intermediates via quantitative mass spectrometric analysis. We found significantly elevated glucose-6-phosphate/glucose (G6P/glucose) ratio compared with saline-treated WT hearts (Figure 6C), consistent with elevated glycolytic flux in the FXN-KO heart. Several metabolic intermediates in glucose utilizing pathways showed a consistent elevation in the FXN-KO heart (Figure 6, D-G), including elevation of metabolite ratio reduced/oxidized glutathione (GSH/GSSG), which is indicative of increased pentose phosphate flux (Figure $6 \mathrm{H})(41,42)$. Together, these data indicate that enhanced glucose flux in this failing heart model is mismatched with mitochondrial metabolism. These data are congruent with the literature, which describes increased cardiac glucose uptake in both the FRDA patient and the FXN-KO mouse (19). Furthermore, this metabolic shift toward glycolysis but incomplete glucose oxidation in mitochondria (Figure 6B) is a consistent feature in HF models of impaired oxidative metabolism (43). Interestingly, NMN supplementation normalized serum lactate levels in the FXN-KO mouse as compared with WT mice (Figure 6A), consistent with the literature, which shows reduced lactic acidosis in NMN-treated exercised mice (44). These data suggest NMN supplementation reduced tissue lactate production or glycolytic flux. Indeed, we observed a significant reduction in the cardiac G6P/glucose ratio upon NMN supplementation (Figure 6C), consistent with significant reductions in glycolytic intermediates of several other glucose metabolites in the FXN-KO heart (Figure 6, D-H). Furthermore, NMN increased metabolite levels in glucose utilizing pathways in the Sirt3 ablated dKO mice (Figure 6, D-H), consistent with the induction in carbohydrate oxidation observed in the NMN-treated SIRT3-KO mouse (Figure 5C). Given these opposing effects of NMN between the FXN-KO and Sirt3-deficient models, these data support an NMN-induced, SIRT3-dependent reduction in glucose metabolism in the FXN-KO heart, which does not appear to be driven by changes in the expression of key proteins involved in glucose uptake, metabolism, and oxidation (Supplemental Figure 5, A-E). Overall, these data show NMN treatment resulted in a SIRT3-dependent decrease in systemic (Figure 5) and cardiac (Figure 6) glucose utilization, thereby enhancing CE and function.

\section{Discussion}

Here, we report SIRT3-dependent restoration of cardiac function and energy metabolism upon NMN supplementation in the FXN-KO failing heart. This investigation was sparked by evidence supporting a redox imbalance (Figure 1B) contributing to pathological, progressive hyperacetylation of mitochondrial proteins (Figure 1A); alterations in mitochondrial $\mathrm{NAD}^{+}$homeostasis (Figure 1, C and D, and Supplemental Figure 1E); and decreased SIRT3 deacetylase activity (Figure 1E and Supplemental Figure 1, G-I). While NMN supplementation boosted $\mathrm{NAD}^{+}$levels in the FXN-KO and $\mathrm{dKO}$ heart failing models (Figure 3B), it did not lead to a sustained restoration in mitochondrial redox imbalance (data not shown), nor did it reduce global hyperacetylation of mitochondrial proteins in these models (Supplemental Figure 2, B and C). These observations are consistent with previous findings, where restoration of $\mathrm{NAD}^{+}$levels via NMN partially, in a complex I-deficient model, normalized the $\mathrm{NADH} / \mathrm{NAD}^{+}$ratio and led to a modest, qualitative decrease in global acetylation (45). In fact, the only model to significantly drive a decrease in acetylation with genetic augmentation of $\mathrm{NAD}^{+}$synthesis did so via overexpression of NAMPT (6), effectively reversing the redoxinduced acetylation. Remarkably, NMN supplementation restored cardiac function and energy metabolism in the FXN-KO heart but required SIRT3 to achieve these improvements, demonstrating that the altered mitochondrial $\mathrm{NAD}^{+}$homeostasis is a critical contributor to the pathogenesis of HF in the FXN-KO mice. Furthermore, these data highlight a pivotal role of key target sites of SIRT3 deacetylase activity, rather than global mitochondrial protein hyperacetylation, in the development of the FXN-KO cardiomyopathy. 
A

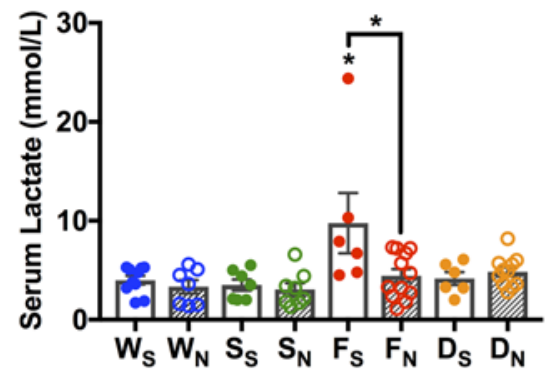

D

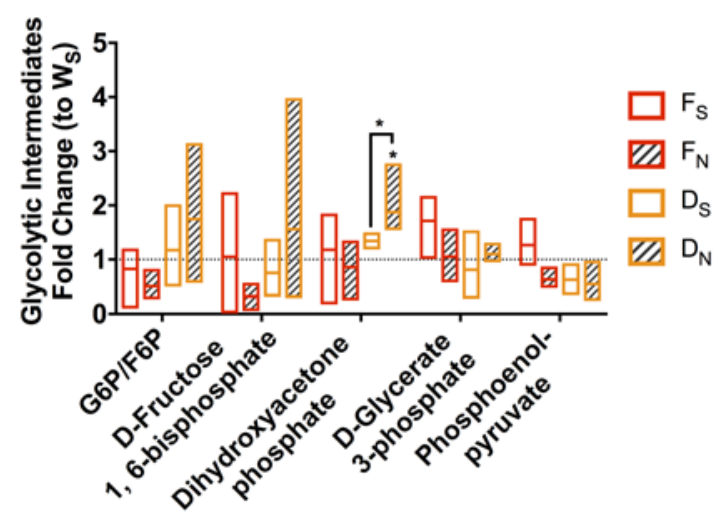

G

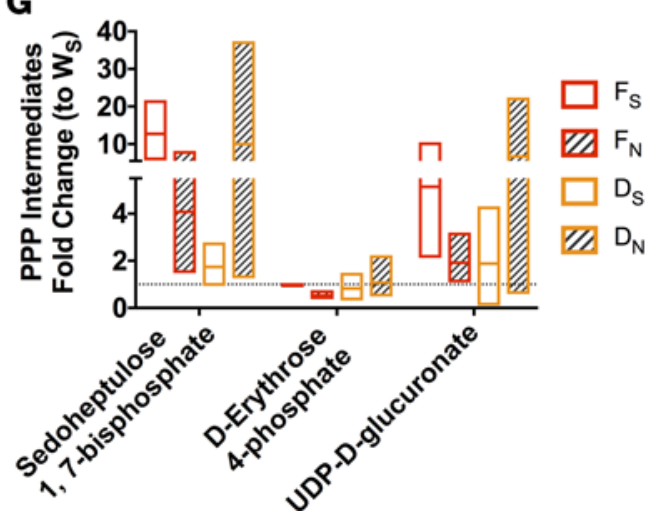

C

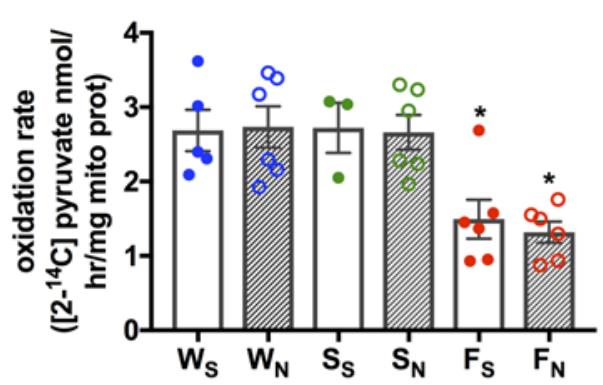

E

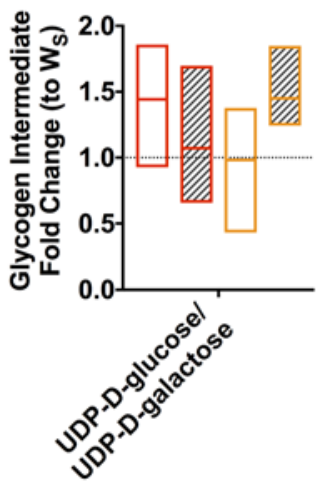

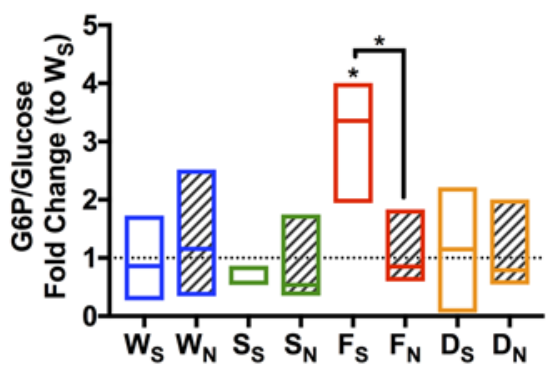

$\mathbf{F}$

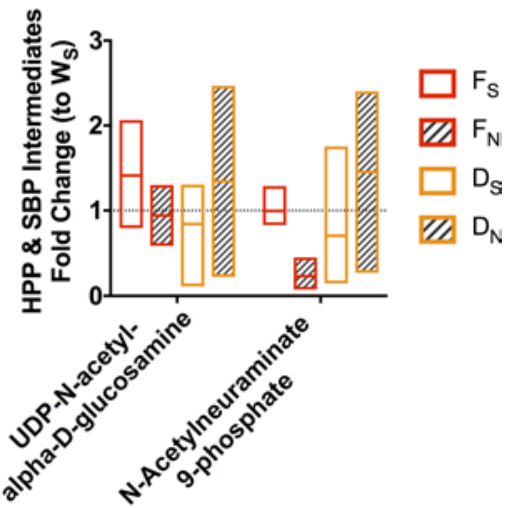

Figure 6. NMN glucose utilization in the FXN-KO mouse in a SIRT3-dependnet manner. (A) Serum lactate levels. Values are means \pm SEM ( $n=6-12$ mice/ group). (B) $2-{ }^{14} \mathrm{C}$-pyruvate oxidation measured in cardiac tissue lysates. Values are means \pm SEM ( $n=3-6$ mice/group). (C-H) Metabolomic profiling from cardiac tissue of metabolites involved in glucose metabolism. Ratio of glucose committed to glucose metabolism in the heart (G6P/glucose ratio, C), glycolysis intermediates (D), glycogen intermediate (E), hexosamine biosynthesis pathway (HBP) and sialic acid biosynthesis pathway (SBP) intermediates $(\mathbf{F})$, pentose phosphate pathway (PPP) intermediates (G), and glutathione/GSSG ratio (H). Bar line represents sample mean $\left(n=3-5\right.$ mice/group). ${ }^{*} P<$ 0.05 , difference from saline-treated WT as determined by two-way ANOVA for 8 groups (4 genotypes, 2 treatment) and Bonferroni correction. W, WT; F, FXN-KO; S, SIRT3-KO; D, dKO; subscript S, saline; and subscript N, NMN.

We and others have postulated that a reduction in the mitochondrial $\mathrm{NAD}^{+}$pool could negatively impact the activity of mitochondrial protein deacetylase SIRT3, which in turn could be contributing to the accumulation of acetylation marks $(5,15)$. Consistent with this notion, a meta-analysis of the SIRT3-KO cardiac acetylome to identify SIRT3 targets suggests reduced and/or insufficient SIRT3 deacetylase activity in the FXN-KO heart (Figure 1, E-F and Supplemental Figure 1, G-I). These data illustrate SIRT3 activity as a potential therapeutic target for regulating key sites of acetylation in the FXN-KO heart. Sirt3 ablation in the SIRT3-KO and dKO mice manifests in the development of cardiac concentric hypertrophy (Figure 2, G and $\mathrm{H})$, consistent with the global SIRT3-KO literature $(9,10)$. We also found a role of SIRT3 for normal contractile function, which may explain the increased susceptibility to cardiac stressors in the global SIRT3-KO 
mouse (9-11). Given that global SIRT3-KO mice only show differences in cardiac function from WT controls upon cardiac stress (9-11), the findings that the FXN-KO and $\mathrm{dKO}$ mice showed no functional differences at baseline are consistent. As such, we predict that cardiac induced-stress in the FXN-KO and dKO mice might reveal an exacerbated maladaptive response to the stress in the dKO model. Lastly, contrary to prior studies of skeletal muscle-specific SIRT3 ablation (32), our heart- and skeletal muscle-specific SIRT3-KO and dKO mice gained more weight overall when compared with WT and FXN-KO mice, respectively (Figure 2F), suggesting that the weight gain in our models is driven by SIRT3 deficiency in the heart. However, future investigations with a cardiac-specific deletion model of Fxn and Sirt3 will be crucial for affirming the heart-autonomous role of SIRT3 in the setting of FRDA HF that we report here.

We found that NMN supplementation in the FXN-KO mice normalized the defective cardiac contractility and diastolic function in this model, as compared with saline-treated WT controls in a SIRT3-dependent manner (Figure 3, E-I). An additional parameter of contractility, ejection time (ET) measured by echocardiography, was shortened in the FXN-KO, SIRT3-KO, and dKO mice, demonstrating impaired contractility (Supplemental Figure 2H). A shortened ET is an indication of impaired contractility in patients with HF (46) and is, in part, a prognostic indicator of severe LV dysfunction in patients with idiopathic-dilated cardiomyopathy (47). NMN significantly increased ET in the FXN-KO model, but not in the SIRT3-KO or dKO models (Supplemental Figure 2H), further evidence of SIRT3-depdendent improvements in contractility. The improvements in contractility and ATP-dependent diastolic function in the NMN-treated FXN-KO mice provide important insight into the molecular mechanisms by which NMN and SIRT3 enhance cardiac function. Myocardial PCr/ATP ratio is a strong predictor of total and cardiovascular mortality in patients with dilated cardiomyopathy (48). NMN increased both PCr/ATP and $\mathrm{PCr} / \mathrm{Cr}$ ratios, likely achieved by improvements in cardiac energy utilization rather than by enhancement of ATP production. Indeed, NMN decreased energy wasting in the FXN-KO heart, but not in the dKO heart (Figure 4F and Supplemental Figure 3, E and F), significantly normalizing energy utilization in a SIRT3-dependent manner. Restoration of energy balance resulted in a complete normalization of $\mathrm{CE}$ and of the VC ratio (Figure 4, C and D, respectively). These improvements in the FXN-KO heart warrant further investigation aimed at maximizing the therapeutic impact of NMN. Furthermore, these improvements serve as important preclinical data that may guide future therapies for FRDA patients.

Notably, the NMN-mediated reduction in energy wasting within the FXN-KO heart paralleled a reduction in whole-body $\mathrm{EE}$, in part achieved via decreased cellular respiration and substrate oxidation (Figure 5). These data reveal therapeutic effects of NMN that are SIRT3 dependent (i.e., shifts in wholebody carbohydrate and cardiac glucose metabolism), as well as SIRT3 independent (i.e., whole-body and cardiac FA metabolism). Whole-body daily FA oxidation was slightly elevated in the FXN-KO (Figure $5 B)$, a trend consistent with the literature in $\operatorname{HF}$ patients $(37,38)$. NMN supplementation drove a statistically significant reduction in FA oxidation in the FXN-KO mouse. Additionally, we found a trending elevation in serum triacylglycerides in FXN-KO mice that was reduced with NMN, along with a reduction in circulating nonesterified FAs (NEFAs) (Supplemental Figure 5, G and H, respectively). These data suggest that NMN reduced global FA availability, a finding consistent with literature that describes reduced serum triacylglycerides and free FAs with long-term administration of NMN in mice (1). Within in the heart, saturated FAs accumulated in the FXN-KO and dKO models (Supplemental Figure 5I), which is also observed in FRDA patients (19). Interestingly, across all genotypes, NMN supplementation drove a trending reduction in FA content, consistent with an overall reduction in FA availability. Furthermore, NMN reduced acyl-carnitine species in the WT and SIRT3-KO hearts and increased these same species in the FXN-KO and dKO failing heart models (Supplemental Figure 5J). Lastly, NMN treatment in WT and SIRT3-KO mice resulted in significantly depressed cardiac FA oxidation rates (Supplemental Figure 5F), as compared with saline-treated WT controls. Overall, these data support an NMN-induced lowering of FA availability and utilization that is independent of SIRT3.

The remarkable decrease in whole-body EE and cardiac energy wasting was achieved by both a SIRT3-independent decrease in FA metabolism and a SIRT3-dependent decrease in carbohydrate metabolism (Figure 5, A-C). These data illustrate a possible peripheral metabolic effect of NMN that could be mediated by cardiac expression of SIRT3 in the FXN-KO. Drugs that have peripheral metabolic activities that benefit cardiac function have been developed for therapy against HF. For example, the partial FA oxidation inhibitor trimetazidine directly acts on FA oxidation but indirectly improves carbohydrate metabolism in HF patients (49). Similar to the effects of NMN in the FXN-KO heart, trimetazidine improves LV 
systolic function, elevates the cardiac PCr/ATP ratio, and decreases cellular acidosis in human patients with congestive or ischemic failing hearts $(37,50-53)$. These effects are achieved by a global reduction in resting EE with a concomitant whole-body and cardiac decrease in FA oxidation and increase in glucose oxidation $(37,50,53)$. Taken together, our data support a similar systemic activity of NMN to induce shifts in substrate metabolism in the FXN-KO, which in part requires cardiac expression of SIRT3. These findings are important for the continued development of drugs that go beyond improving cardiac function, as HF is accompanied by impaired function in peripheral tissues, such as renal insufficiency and insulin resistance (54).

NMN treatment resulted in a SIRT3-dependent decrease in systemic and cardiac glucose utilization (Figure 5C and Figure 6, respectively). The reduction in cardiac glucose metabolism could be responsible for the improvements in $\mathrm{CE}$, as has been shown in I/R injury models (55-57). In I/R, high glycolytic flux for ATP production (Figure 6, C and D) is uncoupled from glucose oxidation (Figure 6B and Supplemental Figure $5 \mathrm{~A}$ ), which leads to excessive lactate production (Figure $6 \mathrm{~A}$ ) and proton generation, promoting acidification of cardiomyocytes and serum. To normalize cytosolic $\mathrm{pH}$, the ATP-dependent sodium-proton exchanger (NHE) exhausts cardiac ATP to pump the excess protons out of the cardiomyocytes and into the extracellular space, which comes at the cost of cardiac contractility and CE (55-57). CE and function is improved by drugs that enhance coupling of glycolysis to glucose oxidation (55), drugs that inhibit NHE during reperfusion (55), or drugs that inhibit glycolytic rates $(58,59)$. Our data suggests that NMN effectively inhibits glycolysis in the FXN-KO heart by reducing glucose availability (Figure 6C), which may partially explain the improved CE in this model (Figure $4 \mathrm{C}$ ). These effects of NMN required cardiac expression of SIRT3 in the FXN-KO. Interestingly, SIRT3 was shown to be a crucial regulator of glucose flux in the skeletal muscle and heart under nutrient-overload conditions (60). SIRT3-KO mice stressed by a high-fat diet show a strong trending decrease $(P=0.065)$ in glucose uptake in the heart, as compared with high-fat-fed WT mice (60). This reduction in glucose uptake was statistically significant in the skeletal muscle of SIRT3-KO mice. Loss of SIRT3 in the skeletal muscle impaired complex formation of mitochondrial proteins voltage-dependent anion channel (VDAC) and adenine nucleotide translocator (ANT), which resulted in decreased association of hexokinase II to the mitochondria, decreased conversion of glucose to G6P, and reduced downstream glucose metabolism $(60,61)$. Given the SIRT3-dependent changes in glucose utilization and metabolism, and their potential role in the normalization of $\mathrm{CE}$, it will be of interest to evaluate a regulatory role of SIRT3 on glucose metabolism within the FXN-KO heart.

In summary, our findings provide mechanistic insight into SIRT3-dependent therapeutic effects of NMN treatment, potentially via deacetylation at key SIRT3 targets in the FXN-KO heart. Recent work from our lab characterized the chemistry of nonenzymatic acylation of common marks like acetylation, as well as novel acyl marks (62). Additionally, recent literature describes high reactivity of lysine residues to nonenzymatic acetylation at strong SIRT3 targets sites, indicating an important role for SIRT3 in suppressing acetylation at these sites rather than at all sites of acetylation $(30,63)$. In these studies, low acetylation stoichiometry at these SIRT3-targeted sites led to the conclusion that SIRT3 is unlikely to regulate protein function with further deacetylation in the context of a hyperacetylated mitochondria (e.g., FXN-KO heart). However, our data define a clear role for SIRT3 in mediating the beneficial effects of NMN in this HF model; thus, future studies in the FXN-KO and dKO models would be of interest to evaluate the consequences of manipulating of redox-induced acetylation versus SIRT3-regulated acetylation. These studies include studies dedicated to varying the NMN dosing regimen, as well as acetyl-proteomic analysis of hearts from these NMN-treated mice. Our findings presented here serve as important preclinical data to highlight NMN supplementation and/or SIRT3 agonist treatment as potential therapeutic strategies in FRDA patients and HF.

\section{Methods}

Animal models and studies. Transgenic mice with ablation of Fxn (FXN-KO) under the control of the MCK-Cre promoter on a mixed C57BL/6J and 129Sv background have been previously characterized (16). In our studies, both alleles were conditional (i.e., $F x n^{\mathrm{L} 3 / \mathrm{L} 3}$ ), rather than the deletion allele/conditional allele model $\left(F x n^{\mathrm{L} 3 / \Delta}\right)$ previously characterized (16). Mouse ES cells were generated containing the floxed SIRT3 gene (SIRT32 ${ }^{\mathrm{L} 2 / \mathrm{L} 2}$ ) on the C57BL/6J strain, as described previously (13). The SIRT3 ${ }^{\mathrm{L} 2 / \mathrm{L} 2}$ and $F x n^{\mathrm{L} 3 / \mathrm{L} 3}$ were crossed to generate the dKO model (SIRT3 $\left.3^{\mathrm{L} 2 / \mathrm{L} 2} / F x n^{\mathrm{L} 3 / \mathrm{L} 3}\right)$, backcrossed on the C57BL/6J background for 3 generations. Using standard mouse genetic techniques, mice containing the floxed 
gene were crossed with the C57BL/6J MCK-Cre mouse to generate homozygous KO mice. All mice used in these studies were male. We injected saline or NMN (Sigma-Aldrich, N3501) i.p. at (500 mg/kg) twice weekly from 6 weeks of age until 10-11 weeks of age. Animals were culled 24 hours after their last injection. Indirect calorimetry was measured as described (64) after mice were acclimated for 5 hours to a 8-chamber CLAMS system (Columbus Instruments).

Noninvasive and invasive cardiac function assessment. Serial echocardiography was performed on conscious mice from all groups with a Vevo 2100 high-resolution imaging system. In vivo PV-loop analysis was performed as previously described (65). See Supplemental Methods for a detailed description of the procedure.

$R N A$ isolation and quantitative PCR ( $q P C R$ ). Total heart RNA was isolated from flash-frozen tissue using the RNeasy Mini Kit (Qiagen). cDNA was synthesized using the Bio-Rad iScript cDNA synthesis kit and diluted 1:10 with nuclease-free water. qPCR reaction mix contained $2.5 \mu 1$ 1:10 cDNA, $0.5 \mu \mathrm{M}$ of $10 \mu \mathrm{M}$ forward and reverse primer mix, $4 \mu \mathrm{l}$ iTAQ Universal SYBR green Supermix (Bio-Rad), and $1 \mu 1$ RNase/ DNase-free water. qPCR was run using a Viia 7 Real-Time PCR system (Applied Biosystems).

Tissue isolation and Western blot analysis. Crude mitochondria were isolated from fresh hearts by differential centrifugation. Western blot analysis was performed with 20-30 $\mu \mathrm{g}$ protein per lane. Membranes were incubated in primary antibody either overnight at $4^{\circ} \mathrm{C}$ or at room temperature for $1-2$ hours with rocking. Primary antibodies were purchased from the following companies: acetylated-lysine (Cell Signaling Technology [CST], 9441); SIRT3 (D22A3; CST, 5490); pAMPKa (Th172; CST, 2535P); AMPKa (CST, 2603P); total OXPHOS (MitoSciences, Abcam, ab110413); pPDH (S293; CST, Calbiochem, AP1062); PDH E1A (Abcam, 1101416); GLUT1 (CST, 12939); GLUT4 (Abcam, ab654-250); and GAPDH (CST, 97166). Western blots were developed using a Li-Cor Odyssey CLx. Blots were quantified using the Li-Cor software.

Gene microarray. Previously published gene microarray data were downloaded from the NCBI GEO repository (GSE31208; ref. 66). Affymetrix Gene Chip microarray data underwent strict quality control processing using the simpleaffy (67) package in the Bioconductor (68) suite from the R statistical programming environment (69). Log-scale robust multiarray analysis (RMA) from the affy package (70) was used for normalization to eliminate systematic differences across the arrays. We then employed a linear regression model with empirical Bayes methods for parameter estimation, using the limma package (71), to calculate differential expression across the conditions. The FDR method was used to control for multiple hypothesis testing. Gene set enrichment analysis (GSEA; ref. 72) was performed to identify enriched molecular functions, cellular pathways, and gene networks in the resulting list of genes whose expression profiles show differences between the conditions.

Quantitative metabolomics. Flash frozen hearts were pulverized and weighed (10 mg). Metabolite extraction was performed ( $500 \mu 1$ solvent/10 mg of tissue) as described in a previous study (73). Ultimate 3000 UHPLC (Dionex) was coupled to Q Exactive Plus mass spectrometer (QE-MS, Thermo Scientific) for metabolite profiling. Liquid chromatography mass spectrometry (LC-MS) peak extraction and integration were performed using commercially available software Sieve 2.2 (Thermo Scientific). See the Supplemental Methods for details.

Quantitative proteomics. Relative changes in protein acetylation and protein abundance were determined by quantitative mass spectrometry as described previously (21). Briefly, mitochondrial proteins were digested with trypsin and the resulting peptides were labeled with 6-plex Tandem Mass Tags (TMT, Thermo Fisher Scientific). For each time point, WT and FXN-KO samples $(n=3)$ were mixed and subjected to IP using PTMScan Acetyl-Lysine Motif antibody (CST). Both the IP eluate and the input fraction were subjected to nanoscale liquid chromatography coupled to tandem mass spectrometry (nanoLC-MS/MS) on a Q Exactive Plus Orbitrap mass spectrometer and data analysis with Proteome Discoverer 2.1 Service Pack 1 (SP1). The mass spectrometry proteomics data have been deposited to the ProteomeXchange Consortium via the PRIDE partner repository with the dataset identifier PXD006754. See the Supplemental Methods for details.

Serum metabolite profiling. Serum triglycerides (TG), total NEFAs, and lactate were measured on a Beckman DxC600 clinical analyzer. Reagents were also from Beckman, except NEFA, from Wako.

Statistics. All statistics performed, except proteomics, were differences from saline-treated WT values by two-way ANOVA and Bonferroni post-hoc for 8 groups (4 genotypes, 2 treatment groups), with significance being defined as $P \leq 0.05$. For proteomics, each TMT 6-plex (WT and FXN-KO at 5, 8, and 13 weeks; $n=3$ /genotype; 1 age group per plex) were difference from WT values by Student's t-test and Benjamini-Hochberg FDR correction, with significance being defined as $P_{\text {adjusted }} \leq 0.05$. 
Study approval. All animal studies were carried out under protocols approved by the Duke University Animal Care and Use Committee (protocol A091-17-04). Animal housing, care, and husbandry were overseen by the Duke Department of Laboratory Animal Resources, which is accredited by the Association for Assessment and Accreditation of Laboratory Animal Care (AAALAC).

\section{Author contributions}

Conceptualization was completed by ASM, DMA, and MDH. Investigation was completed by ASM, DMA, KAH, LM, JL, XL MJM, DPB, and PAG. Writing was completed by ASM and MDH. Review and editing were completed by ASM, DMA, KAH, DPB, LM, HC, JL, XL, MJM, PAG, JWL, RMP, and $\mathrm{MDH}$. MDH supervised the project. Project administration was provided by $\mathrm{MDH}$. Funding acquisition was provided by ASM and $\mathrm{MDH}$.

\section{Acknowledgments}

We would like to thank Tim Koves and Deborah Muoio for use of the Comprehensive Lab Animal Monitoring System (CLAMS); we thank David Corcocan for GSEA and computational support; we thank Hélène Puccio for sharing the FXN-KO mouse model; we thank the entire Hirschey lab for their feedback, as well as members of the Duke Molecular Physiology Institute, specifically Scott Crown and James Draper; we thank Chris Newgard, Howard Rockman, and other members of Angelical S. Martin's dissertation committee for feedback. We would also like to acknowledge funding support from Friedreich's Ataxia Research Alliance (FARA); the American Heart Association grants 12SDG8840004 and 12IRG9010008; the NIH NIAAA grant R01AA022146; the National Institute of Diabetes, Digestive, and Kidney Diseases of the NIH under award number P30DK096493; and the Mandel Center for Hypertension and Atherosclerosis at Duke. ASM was supported by an NIH/NIGMS training grant to Duke University Pharmacological Sciences Training Program (5T32GM007105-40) and is supported by an NIH predoctoral fellowship (1F31HL123275-01); DMA is supported by 1K08HL125905-01. The funding sources had no role in study design, data collection and analysis, decision to publish, or preparation of the manuscript.

Address for correspondence: Matthew Hirschey, Duke Molecular Physiology Institute, and Sarah W. Stedman Nutrition and Metabolism Center, Duke University, 300 N Duke Street, Durham, North Carolina 27701, USA. Phone: 919.479.2315; Email: matthew.hirschey@duke.edu.

1. Mills KF, et al. Long-Term Administration of Nicotinamide Mononucleotide Mitigates Age-Associated Physiological Decline in Mice. Cell Metab. 2016;24(6):795-806.

2. Trammell SA, et al. Nicotinamide riboside is uniquely and orally bioavailable in mice and humans. Nat Commun. 2016;7:12948.

3. Tran MT, et al. PGC1 $\alpha$ drives NAD biosynthesis linking oxidative metabolism to renal protection. Nature. 2016;531(7595):528-532.

4. Cantó $\mathrm{C}$, et al. The $\mathrm{NAD}(+)$ precursor nicotinamide riboside enhances oxidative metabolism and protects against high-fat diet-induced obesity. Cell Metab. 2012;15(6):838-847.

5. Hershberger KA, Martin AS, Hirschey MD. Role of $\mathrm{NAD}(+)$ and mitochondrial sirtuins in cardiac and renal diseases. Nat Rev Nephrol. 2017;13(4):213-225.

6. Lee CF, et al. Normalization of NAD+ Redox Balance as a Therapy for Heart Failure. Circulation. 2016;134(12):883-894

7. Xu W, Barrientos T, Mao L, Rockman HA, Sauve AA, Andrews NC. Lethal Cardiomyopathy in Mice Lacking Transferrin Receptor in the Heart. Cell Rep. 2015;13(3):533-545.

8. Yamamoto T, Byun J, Zhai P, Ikeda Y, Oka S, Sadoshima J. Nicotinamide mononucleotide, an intermediate of NAD+ synthesis, protects the heart from ischemia and reperfusion. PLoS One. 2014;9(6):e98972.

9. Pillai VB, et al. Exogenous NAD blocks cardiac hypertrophic response via activation of the SIRT3-LKB1-AMP-activated kinase pathway. J Biol Chem. 2010;285(5):3133-3144.

10. Hafner AV, et al. Regulation of the mPTP by SIRT3-mediated deacetylation of CypD at lysine 166 suppresses age-related cardiac hypertrophy. Aging (Albany NY). 2010;2(12):914-923.

11. Porter GA, Urciuoli WR, Brookes PS, Nadtochiy SM. SIRT3 deficiency exacerbates ischemia-reperfusion injury: implication for aged hearts. Am J Physiol Heart Circ Physiol. 2014;306(12):H1602-H1609.

12. Ahn BH, et al. A role for the mitochondrial deacetylase Sirt3 in regulating energy homeostasis. Proc Natl Acad Sci USA. 2008;105(38):14447-14452.

13. Lombard DB, et al. Mammalian Sir2 homolog SIRT3 regulates global mitochondrial lysine acetylation. Mol Cell Biol. 2007;27(24):8807-8814.

14. Xu W, Barrientos T, Mao L, Rockman HA, Sauve AA, Andrews NC. Lethal Cardiomyopathy in Mice Lacking Transferrin Receptor in the Heart. Cell Rep. 2015;13(3):533-545.

15. Horton JL, et al. Mitochondrial protein hyperacetylation in the failing heart. JCI Insight. 2016;2(1):e84897.

16. Puccio H, et al. Mouse models for Friedreich ataxia exhibit cardiomyopathy, sensory nerve defect and Fe-S enzyme deficiency 
followed by intramitochondrial iron deposits. Nat Genet. 2001;27(2):181-186.

17. Wagner GR, Pride PM, Babbey CM, Payne RM. Friedreich's ataxia reveals a mechanism for coordinate regulation of oxidative metabolism via feedback inhibition of the SIRT3 deacetylase. Hum Mol Genet. 2012;21(12):2688-2697.

18. Koeppen AH. Friedreich's ataxia: pathology, pathogenesis, and molecular genetics. J Neurol Sci. 2011;303(1-2):1-12.

19. Payne RM, et al. Fatty acid oxidation is disrupted in the FRDA heart. Presented at: International Ataxia Research Conference; March 28, 2015; Windsor, United Kingdom. Session 6.

20. Perdomini M, et al. Prevention and reversal of severe mitochondrial cardiomyopathy by gene therapy in a mouse model of Friedreich's ataxia. Nat Med. 2014;20(5):542-547.

21. McDonnell E, et al. Lipids Reprogram Metabolism to Become a Major Carbon Source for Histone Acetylation. Cell Rep. 2016;17(6):1463-1472.

22. Krebs HA, Gascoyne T. The redox state of the nicotinamide-adenine dinucleotides in rat liver homogenates. Biochem J. 1968;108(4):513-520

23. Pillai JB, Isbatan A, Imai S, Gupta MP. Poly(ADP-ribose) polymerase-1-dependent cardiac myocyte cell death during heart failure is mediated by NAD+ depletion and reduced Sir2alpha deacetylase activity. J Biol Chem. 2005;280(52):43121-43130

24. Xiao CY, et al. Poly(ADP-Ribose) polymerase promotes cardiac remodeling, contractile failure, and translocation of apoptosis-inducing factor in a murine experimental model of aortic banding and heart failure. J Pharmacol Exp Ther. 2005;312(3):891-898

25. Pacher P, Szabó C. Role of poly(ADP-ribose) polymerase 1 (PARP-1) in cardiovascular diseases: the therapeutic potential of PARP inhibitors. Cardiovasc Drug Rev. 2007;25(3):235-260.

26. Cai Y, et al. Nmnat2 protects cardiomyocytes from hypertrophy via activation of SIRT6. FEBS Lett. 2012;586(6):866-874.

27. Ratajczak J, et al. NRK1 controls nicotinamide mononucleotide and nicotinamide riboside metabolism in mammalian cells. Nat Commun. 2016;7:13103.

28. Dittenhafer-Reed KE, et al. SIRT3 mediates multi-tissue coupling for metabolic fuel switching. Cell Metab. 2015;21(4):637-646.

29. Rauniyar N, Yates JR. Isobaric labeling-based relative quantification in shotgun proteomics. J Proteome Res. 2014;13(12):5293-5309.

30. Weinert BT, Moustafa T, Iesmantavicius V, Zechner R, Choudhary C. Analysis of acetylation stoichiometry suggests that SIRT3 repairs nonenzymatic acetylation lesions. EMBO J. 2015;34(21):2620-2632.

31. Alrob OA, et al. Obesity-induced lysine acetylation increases cardiac fatty acid oxidation and impairs insulin signalling. Cardiovasc Res. 2014;103(4):485-497.

32. Fernandez-Marcos PJ, et al. Muscle or liver-specific Sirt3 deficiency induces hyperacetylation of mitochondrial proteins without affecting global metabolic homeostasis. Sci Rep. 2012;2:425

33. Weidemann F, et al. Cardiomyopathy of Friedreich ataxia. J Neurochem. 2013;126 Suppl 1:88-93.

34. Suga H. Ventricular energetics. Physiol Rev. 1990;70(2):247-277.

35. Szabó G, et al. Ventricular energetics after the Fontan operation: contractility-afterload mismatch. J Thorac Cardiovasc Surg. 2003;125(5):1061-1069

36. Walley KR. Left ventricular function: time-varying elastance and left ventricular aortic coupling. Crit Care. 2016;20:270.

37. Fragasso G, et al. Effect of partial inhibition of fatty acid oxidation by trimetazidine on whole body energy metabolism in patients with chronic heart failure. Heart. 2011;97(18):1495-1500.

38. Lommi J, Kupari M, Yki-Järvinen H. Free fatty acid kinetics and oxidation in congestive heart failure. Am J Cardiol. 1998;81(1):45-50.

39. Even PC, Nadkarni NA. Indirect calorimetry in laboratory mice and rats: principles, practical considerations, interpretation and perspectives. Am J Physiol Regul Integr Comp Physiol. 2012;303(5):R459-R476.

40. Speakman JR. Measuring energy metabolism in the mouse - theoretical, practical, and analytical considerations. Front Physiol. 2013;4:34.

41. Kolwicz SC, Tian R. Glucose metabolism and cardiac hypertrophy. Cardiovasc Res. 2011;90(2):194-201.

42. Doenst T, Nguyen TD, Abel ED. Cardiac metabolism in heart failure: implications beyond ATP production. Circ Res. 2013;113(6):709-724

43. Kolwicz SC, Purohit S, Tian R. Cardiac metabolism and its interactions with contraction, growth, and survival of cardiomyocytes. Circ Res. 2013;113(5):603-616.

44. Frederick DW, et al. Loss of NAD Homeostasis Leads to Progressive and Reversible Degeneration of Skeletal Muscle. Cell Metab. 2016;24(2):269-282.

45. Karamanlidis G, et al. Mitochondrial complex I deficiency increases protein acetylation and accelerates heart failure. Cell Metab. 2013;18(2):239-250

46. Weissler AM, Peeler RG, Roehll WH. Relationships between left ventricular ejection time, stroke volume, and heart rate in normal individuals and patients with cardiovascular disease. Am Heart J. 1961;62:367-378.

47. Dujardin KS, Tei C, Yeo TC, Hodge DO, Rossi A, Seward JB. Prognostic value of a Doppler index combining systolic and diastolic performance in idiopathic-dilated cardiomyopathy. Am J Cardiol. 1998;82(9):1071-1076.

48. Neubauer S, et al. Myocardial phosphocreatine-to-ATP ratio is a predictor of mortality in patients with dilated cardiomyopathy. Circulation. 1997;96(7):2190-2196.

49. Inamdar AA, Inamdar AC. Heart Failure: Diagnosis, Management and Utilization. J Clin Med. 2016;5(7):E62.

50. Fragasso G, et al. Effect of partial fatty acid oxidation inhibition with trimetazidine on mortality and morbidity in heart failure: results from an international multicentre retrospective cohort study. Int J Cardiol. 2013;163(3):320-325.

51. Nickel A, Löffler J, Maack C. Myocardial energetics in heart failure. Basic Res Cardiol. 2013;108(4):358.

52. Gunes Y, Guntekin U, Tuncer M, Sahin M. Improved left and right ventricular functions with trimetazidine in patients with heart failure: a tissue Doppler study. Heart Vessels. 2009;24(4):277-282.

53. Chrusciel P, Rysz J, Banach M. Defining the role of trimetazidine in the treatment of cardiovascular disorders: some insights on its role in heart failure and peripheral artery disease. Drugs. 2014;74(9):971-980.

54. Brown DA, et al. Expert consensus document: Mitochondrial function as a therapeutic target in heart failure. Nat Rev Cardiol. 2017;14(4):238-250

55. Liu B, Clanachan AS, Schulz R, Lopaschuk GD. Cardiac efficiency is improved after ischemia by altering both the source and 
fate of protons. Circ Res. 1996;79(5):940-948.

56. Hata K, Takasago T, Saeki A, Nishioka T, Goto Y. Stunned myocardium after rapid correction of acidosis. Increased oxygen cost of contractility and the role of the $\mathrm{Na}(+)-\mathrm{H}+$ exchange system. Circ Res. 1994;74(5):794-805

57. Lee L, Horowitz J, Frenneaux M. Metabolic manipulation in ischaemic heart disease, a novel approach to treatment. Eur Heart J. 2004;25(8):634-641.

58. Finegan BA, Clanachan AS, Coulson CS, Lopaschuk GD. Adenosine modification of energy substrate use in isolated hearts perfused with fatty acids. Am J Physiol. 1992;262(5 Pt 2):H1501-H1507.

59. Finegan BA, Lopaschuk GD, Coulson CS, Clanachan AS. Adenosine alters glucose use during ischemia and reperfusion in isolated rat hearts. Circulation. 1993;87(3):900-908.

60. Lantier L, et al. SIRT3 Is Crucial for Maintaining Skeletal Muscle Insulin Action and Protects Against Severe Insulin Resistance in High-Fat-Fed Mice. Diabetes. 2015;64(9):3081-3092.

61. Fueger PT, et al. Hexokinase II partial knockout impairs exercise-stimulated glucose uptake in oxidative muscles of mice. Am $J$ Physiol Endocrinol Metab. 2003;285(5):E958-E963.

62. Wagner GR, et al. A Class of Reactive Acyl-CoA Species Reveals the Non-enzymatic Origins of Protein Acylation. Cell Metab. 2017;25(4):823-837.e8.

63. Weinert BT, Satpathy S, Hansen BK, Lyon D, Jensen LJ, Choudhary C. Accurate Quantification of Site-specific Acetylation Stoichiometry Reveals the Impact of Sirtuin Deacetylase CobB on the E. coli Acetylome. Mol Cell Proteomics. 2017;16(5):759-769.

64. Albarado DC, et al. Impaired coordination of nutrient intake and substrate oxidation in melanocortin-4 receptor knockout mice. Endocrinology. 2004;145(1):243-252.

65. Yoo B, et al. Beta1-adrenergic receptors stimulate cardiac contractility and CaMKII activation in vivo and enhance cardiac dysfunction following myocardial infarction. Am J Physiol Heart Circ Physiol. 2009;297(4):H1377-H1386.

66. Huang ML, Becker EM, Whitnall M, Suryo Rahmanto Y, Ponka P, Richardson DR. Elucidation of the mechanism of mitochondrial iron loading in Friedreich's ataxia by analysis of a mouse mutant. Proc Natl Acad Sci USA. 2009;106(38):16381-16386.

67. Wilson CL, Miller CJ. Simpleaffy: a BioConductor package for Affymetrix Quality Control and data analysis. Bioinformatics. 2005;21(18):3683-3685.

68. Gentleman RC, et al. Bioconductor: open software development for computational biology and bioinformatics. Genome Biol. 2004;5(10):R80

69. R Core Team. R: A language and environment for statistical computing. R Foundation for Statistical Computing, Vienna, Austria. http://www.R-project.org/. Accessed June 9, 2017.

70. Gautier L, Cope L, Bolstad BM, Irizarry RA. affy--analysis of Affymetrix GeneChip data at the probe level. Bioinformatics. 2004;20(3):307-315.

71. Smyth GK. LIMMA: linear models for microarray data. In: Genlteman R, Carey V, Huber W, Irizarry R, Dudoit S, eds. Bioinformatics and Computational Biology Solutions Using R and Bioconductor. New York, NY: Springer-Verlag New York;2005:397-420.

72. Mootha VK, et al. PGC-1alpha-responsive genes involved in oxidative phosphorylation are coordinately downregulated in human diabetes. Nat Genet. 2003;34(3):267-273.

73. Liu X, et al. High-Resolution Metabolomics with Acyl-CoA Profiling Reveals Widespread Remodeling in Response to Diet. Mol Cell Proteomics. 2015;14(6):1489-1500. 